Scientific paper

\title{
Synthesis, Antimicrobial Activity and in silico Studies on Thymol Esters
}

\author{
Jelena Lazarević,,$^{*}$ Ana Kolarević, ${ }^{2}$ Aleksandra Đorđević, ${ }^{3}$ Gordana Stojanović, ${ }^{3}$ \\ Andrija Šmelcerović, ${ }^{1,2}$ Pierangela Ciuffreda ${ }^{4}$ and Enzo Santaniello ${ }^{5}$ \\ ${ }^{1}$ Department of Chemistry, Faculty of Medicine, University of Niš, Bul. dr Zorana Đinđića 81, 18000 Niš (Serbia) \\ ${ }^{2}$ Department of Pharmacy, Faculty of Medicine, University of Nǐ̌, Bul. dr Zorana Đinđića 81, 18000 Niš (Serbia) \\ ${ }^{3}$ Department of Chemistry, Faculty of Science and Mathematics, University of Niš, Višegradska 33, 18000 Nǐs (Serbia) \\ ${ }^{4}$ Dipartimento di Scienze Biomediche e Cliniche »L. Sacco«, Università degli Studi di Milano, \\ Via G. B. Grassi 74, 20157 Milano, Italy
}

${ }^{5}$ Department of Biomedical Sciences, Humanitas University, Via Manzoni 113, 20089 Rozzano - Milano, Italy

* Corresponding author: E-mail: jelena217@yahoo.com

Received: 11-03-2017

\begin{abstract}
Derivatisation of parent structure in terpenoids often results in enhancement of biological activity of newly obtained compounds. Thymol, a naturally occurring phenol biosynthesized through the terpene pathway, is a well known biocide with strong antimicrobial attributes and diverse therapeutic activities. We have aimed our study on a single modification of phenolic functionality in thymol in order to obtain a small focused library of twenty thymyl esters, ten of which were new compounds. All compounds were involved in in vitro antimicrobial testing. Another important aspect of current study was implementation of in silico calculation of physico-chemical, pharmacokinetic and toxicological properties, which could be helpful by giving an additional guidance in further research.
\end{abstract}

Keywords: chemical synthesis, thymyl esters, in vitro antimicrobial activity, in silico calculation

\section{Introduction}

Terpenoids constitute an abundant and potent group of natural products, which play an important role in the enzyme systems of plants and reflect conspicuous biological activity against various pests. Their biological activity is believed to be related to the nature and the position of functional groups or substituents. ${ }^{1}$ Chemical modification of natural monoterpenoids has been reported to result in enhancement of biological activities when compared to parent compounds. ${ }^{2-5}$ Thymol (1), a monoterpene biosynthetically directly related to para-cymene $(\mathbf{1 a})^{6}$ and a naturally occurring phenol, is a well known biocide and most dominant constituent of the oils of thyme (Thymus vulgaris $\mathrm{L}$.) and oregano (Origanum vulgare $\mathrm{L}$.).

Numerous studies have demonstrated the antimicrobial effect of thymol, ranging from inducing antibiotic

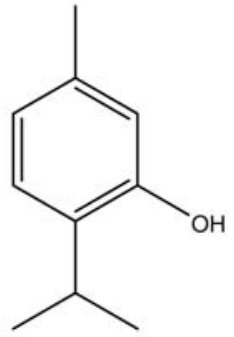

1

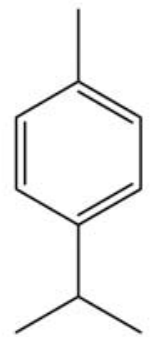

1a
Sheme 1. Chemical structures of thymol (1) and para-cymene (1a).

susceptibility in drug-resistant pathogens through a synergistic effect, to a powerful antioxidant properties. ${ }^{7}$ Thymol has been shown to be an effective fungicide, particularly against fluconazole-resistant strains ${ }^{8}$ and was demonstrated to have strong antimutagenic effect. ${ }^{9}$ In addi- 
tion, there is evidence that thymol has antitumor properties. ${ }^{10}$

The primary mode of antibacterial action of thymol is not fully known, but is believed to involve outer- and inner membrane disruption, and interaction with membrane proteins and intracellular targets. ${ }^{1-16}$ The mode of action of thymol against yeast and fungi has been sparsely investigated, though certain studies point to interactions with the cell envelope and intracellular targets. ${ }^{16-19}$

Thymyl derivatives are well represented in Asteraceae plants, particularly within tribes Senecioneae, Eupatorieae, Inuleae and Helenieae. In some Inula, Doronicum and Pulicaria species, thymyl derivatives rather than sesquiterpenoids are the major root constituents. ${ }^{20-23} \mathrm{~A}$ review by Talavera-Aleman and collaborators ${ }^{24}$ estimated that only $10 \%$ of known functionalized thymyl derivatives have been employed in biological testing, showing vast array of diverse activities, such as antimicrobial ${ }^{4,5,22,25-27}$ (several papers reporting inhibitory activity against plants' pathogenic fungi ${ }^{22,26}$, antioxidant, ${ }^{28}$ antinociceptive, ${ }^{29}$ anti-parasitic (antileishmanial), ${ }^{30,31}$ antiprotozoal, ${ }^{32}$ insecticidal $^{33}$ and piscicidal ${ }^{34}$ activity. The usefulness of thymyl derivatives as transdermal drug delivery enhancers has also been reported. ${ }^{35}$

There are several papers reporting isolation, synthesys $^{4,23,29,33,36}$ and biological activity ${ }^{4,5,22,25,28-30,33}$ of thymyl esters. Grodnitzky and Coats ${ }^{33}$ have tested insecticidal activity of thymyl esters of acetic, dichloracetic, trichloroacetic, chlorodifluoroacetic, pivalic and chloropivalic acid on Musca domestica. Mathela and collaborators $^{4}$ evaluated antibacterial activity of thymyl esters of acetic, propanoic, 2-methylpropanoic, 3-methylbutanoic, but-2-enoic, benzoic and 2-phenylacetic acids and reported the enhancement in the activity of derivatives in comparison to thymol. Kumbhar and Dewang ${ }^{5}$ tested antifungal activity and observed structure-activity relationship, stating that thymyl ethers showed better antifungal potency over esters (thymyl acetate, benzoate, cinnamate, dithymyl malonate, succinate and glutarate) and that the addition of a methylene group or a carbon, an olefinic bond or an aromatic moiety in side chain led to compounds with improved potency over the parent compound (thymol). ${ }^{5}$ Angeles-Lopez et al. ${ }^{29}$ have undertaken a study to establish the potential acute toxicity and the antinociceptive activity in animal models of thymyl esters of $\mathrm{C}_{2}-\mathrm{C}_{6}$ straight chain, $\mathrm{C}_{4}-\mathrm{C}_{6}$ acids positional isomers, diasteroisomers of 2-pentenoic, and of benzoic acid, reporting several esters with antinociceptive effect at a dose of $1 \mathrm{mg} / \mathrm{kg}$.

Keeping in view diverse pharmacological activities of thymol ${ }^{7-19}$ and preliminary results on bioactivity of thymyl derivatives ${ }^{4,5,22,25,28-30,33}$ we have set the aim of our study. By making a single modification of a phenolic functionality in thymol we have obtained a series of ester compounds $(\mathbf{3 a}-\mathbf{t})$, performed their structural characterization, in vitro antimicrobial testing and in silico calcula- tion of physico-chemical, pharmacokinetic and toxicological properties. The most important contribution of this study is the synthesis of ten new compounds (3i, $\mathbf{3 k}-\mathbf{s})$ followed by results obtained in antimicrobial assay and in silico calculations, which all together could be an important aspect and an additional guidance in further research.

\section{Materials and Methods}

\section{1. Chemicals Used}

All of the reagents, standards and solvents used were of analytical reagent grade. Unless specified otherwise, all chemicals were purchased from Merck (Darmstadt, Germany).

\section{2. General Synthetic Procedures}

Acetyl, benzoyl and palmitoyl chloride were purchased from Sigma-Aldrich. The conversion of the other carboxylic acids to acyl chlorides ${ }^{37,38}$ and the preparation of thymyl esters utilized methods from the literature. ${ }^{39,40}$ Scheme 1 presents the synthesis of thymyl esters.

\section{2. General Procedures for Synthesis of Acid Chlorides}

(a) An old procedure developed by Brown ${ }^{37}$ was used for the preparation of volatile acid chlorides. This procedure involves the action of a relatively slightly volatile benzoyl chloride upon an organic acid. Following this protocol, the synthesis of chlorides up to 10 carbons, as well as 2-chloroacetyl chloride was achieved. In a roundbottom flask equipped by a fractionating column $0.25 \mathrm{~mol}$ of the acid and 0.375 mol of benzoyl chloride were placed The mixture was heated until the boiling point was reached, and then the acid chloride was distilled from the reaction mixture. The material so obtained was used directly in the synthesis.

(b) For the preparation of chlorides higher than $10 \mathrm{C}$ atoms the corresponding acid was refluxed for $2 \mathrm{~h}$ with thionyl chloride in $\mathrm{CCl}_{4}{ }^{38}$ The solvent and the excess of thionyl chloride were removed with the aid of the water pump vacuum.

The products obtained in either way were used directly in the synthesis.

\section{2. 2. General Procedure for the Synthesis of Thymyl Esters 3a-t}

Series of thymyl esters were made following the procedure described by Paolini et al. ${ }^{39}$ A solution of acid chloride $(4.5 \mathrm{mmol})$ in $\mathrm{CH}_{2} \mathrm{Cl}_{2}(15 \mathrm{~mL})$ was added drop by drop to a cooled mixture $\left(0{ }^{\circ} \mathrm{C}\right)$ of thymol $(3.3 \mathrm{mmol})$ and $\mathrm{Et}_{3} \mathrm{~N}(4.5 \mathrm{mmol})$ in $\mathrm{CH}_{2} \mathrm{Cl}_{2}(20 \mathrm{~mL})$. The mixture was stirred at room temperature and then refluxed for $3 \mathrm{~h}$. The 
organic layer was washed with water $(3 \times 200 \mathrm{~mL})$, dried over anhydrous $\mathrm{Na}_{2} \mathrm{SO}_{4}$ and concentrated under vacuum. The esters were purified by column chromatography, stationary phase Silica Gel 60 (70-230 mesh), mobile phase (hexane/diethyl ether, gradient 9:1 to 8:2). For yields, see supplementary data.

\section{Thymyl Acetate (3a)}

Chromatographic purification gave colorless oil. $\mathrm{C}_{12} \mathrm{H}_{16} \mathrm{O}_{2}(M=192.25)$; yield 85\%; MS (EI): $m / z(\%) 192$ $\left(\mathrm{M}^{+}\right)$(11.3), 151 (4.8), 150 (42.6), 151 (4.8), 135 (100), 136 (11.0), 115 (7.3), 105 (4.7), 77 (5.1), 43 (4.1); RI (HP5-MS): $1367 ;{ }^{1} \mathrm{H}$ NMR (500.13 MHz, $\left.\mathrm{CDCl}_{3}\right): \delta$ (ppm) $7.22(1 \mathrm{H}, \mathrm{d}, J=8 \mathrm{~Hz}, \mathrm{Ar}-\mathrm{H}), 7.05(1 \mathrm{H}, \mathrm{d}, J=8 \mathrm{~Hz}$, Ar-H), 6.84 (1H, s, Ar-H), 3.00 (1H, spt, $J=7 \mathrm{~Hz}, \mathrm{CH})$, $2.34\left(6 \mathrm{H}, \mathrm{s}, \mathrm{CH}_{3}\right), 1.22\left(6 \mathrm{H}, \mathrm{d}, J=7 \mathrm{~Hz}, \mathrm{CH}_{3}\right) ;{ }^{13} \mathrm{C} \mathrm{NMR}$ $\left(125.76 \mathrm{MHz}, \mathrm{CDCl}_{3}\right): \delta(\mathrm{ppm}) 169.77(\mathrm{C}=\mathrm{O}), 147.84$ $\left(\mathrm{C}_{\mathrm{Ar}}\right), 136.95\left(\mathrm{C}_{\mathrm{Ar}}\right), 136.52\left(\mathrm{C}_{\mathrm{Ar}}\right), 127.52\left(\mathrm{C}_{\mathrm{Ar}}\right), 126.78$ $\left(\mathrm{C}_{\mathrm{Ar}}\right), 123.23\left(\mathrm{C}_{\mathrm{Ar}}\right), 26.89(\mathrm{CH}), 26.57\left(\mathrm{CO}-\mathrm{CH}_{3}\right), 22.99$ $\left(2 \times \mathrm{CH}_{3}\right), 21.21\left(\mathrm{CH}_{3}\right)$.

\section{Thymyl 2-Chloroacetate (3b)}

Chromatographic purification gave colorless oil. $\mathrm{C}_{12} \mathrm{H}_{15} \mathrm{O}_{2} \mathrm{Cl}(M=226.70)$; yield 85\%; MS (EI): $\mathrm{m} / \mathrm{z}(\%)$ $226\left(\mathrm{M}^{+}\right)$(10.1), 150 (30.4), 149 (19.4), 136 (9.4), 133 (11.1), 115 (6.5), 105 (8.1), 91 (14.6), 77 (11.4); RI (HP5MS): $1572 ;{ }^{1} \mathrm{H}$ NMR $\left(500.13 \mathrm{MHz}, \mathrm{CDCl}_{3}\right): \delta(\mathrm{ppm}) 7.24$ $(1 \mathrm{H}, \mathrm{d}, J=8 \mathrm{~Hz}, \mathrm{Ar}-\mathrm{H}), 7.08(1 \mathrm{H}, \mathrm{d}, J=7.6 \mathrm{~Hz}, \mathrm{Ar}-\mathrm{H})$, $6.87(1 \mathrm{H}, \mathrm{s}, \mathrm{Ar}-\mathrm{H}), 4.33\left(2 \mathrm{H}, \mathrm{s}, \mathrm{CH}_{2}\right), 3.00(1 \mathrm{H}, \mathrm{spt}, J=$ $6.90 \mathrm{~Hz}, \mathrm{CH}), 2.34\left(3 \mathrm{H}, \mathrm{s}, \mathrm{CH}_{3}\right), 1.22(6 \mathrm{H}, \mathrm{d}, J=6.90 \mathrm{~Hz}$, $\left.\mathrm{CH}_{3}\right) .{ }^{13} \mathrm{C} \mathrm{NMR}\left(125.76 \mathrm{MHz}, \mathrm{CDCl}_{3}\right): \delta$ (ppm) 166.11 $(\mathrm{C}=\mathrm{O}), 147.45\left(\mathrm{C}_{\mathrm{Ar}}\right), 136.81\left(\mathrm{C}_{\mathrm{Ar}}\right), 136.79\left(\mathrm{C}_{\mathrm{Ar}}\right), 127.64$ $\left(\mathrm{C}_{\mathrm{Ar}}\right), 126.60\left(\mathrm{C}_{\mathrm{Ar}}\right), 122.20\left(\mathrm{C}_{\mathrm{Ar}}\right), 40.74\left(\mathrm{CH}_{2}\right), 27.01$ $(\mathrm{CH}), 22.98\left(2 \times \mathrm{CH}_{3}\right), 20.77\left(\mathrm{CH}_{3}-\mathrm{Ar}\right)$.

\section{Thymyl Propanoate (3c)}

Chromatographic purification gave colorless oil. $\mathrm{C}_{13} \mathrm{H}_{18} \mathrm{O}_{2}(M=206.28)$; yield 82\%; MS (EI): $m / z$ (\%) 206 $\left(\mathrm{M}^{+}\right)$(9.5), 151 (4.5), 150 (42.1), 135 (100), 136 (9.5), 115 (5.7), 105 (5.4), 91 (11.0), 77 (5.1), 57 (12.6); RI (HP5-MS): $1455 ;{ }^{1} \mathrm{H}$ NMR (500.13 $\left.\mathrm{MHz} \mathrm{CDCl}_{3}\right): \delta$ (ppm) $7.21(1 \mathrm{H}, \mathrm{d}, J=8 \mathrm{~Hz}, \mathrm{Ar}-\mathrm{H}), 7.04(1 \mathrm{H}, \mathrm{d}, J=8 \mathrm{~Hz}$, Ar-H), 6.83 (1H, s, Ar-H), 2.99 (1H, spt, $J=14$ Hz, CH), $2.63\left(2 \mathrm{H}, \mathrm{q}, J=8 \mathrm{~Hz}, \mathrm{CH}_{2}\right), 2.33\left(3 \mathrm{H}, \mathrm{s}, \mathrm{CH}_{3}\right), 1.32(3 \mathrm{H}$, $\left.\mathrm{m}, \mathrm{CH}_{3}\right), 1.21\left(6 \mathrm{H}, \mathrm{d}, J=7 \mathrm{~Hz}, \mathrm{CH}_{3}\right) ;{ }^{13} \mathrm{C} \mathrm{NMR}(125.76$ $\left.\mathrm{MHz}, \mathrm{CDCl}_{3}\right): \delta(\mathrm{ppm}) 173.16(\mathrm{C}=\mathrm{O}), 147.93\left(\mathrm{C}_{\mathrm{Ar}}\right)$, $136.96\left(\mathrm{C}_{\mathrm{Ar}}\right), 136.5\left(\mathrm{C}_{\mathrm{Ar}}\right), 126.69\left(\mathrm{C}_{\mathrm{Ar}}\right), 123.24\left(\mathrm{C}_{\mathrm{Ar}}\right)$, $122.77\left(\mathrm{C}_{\mathrm{Ar}}\right), 27.73(\mathrm{CH}), 26.91\left(\mathrm{CH}_{2}\right), 22.90\left(\mathrm{CH}_{3}\right)$, $23.03\left(2 \times \mathrm{CH}_{3}\right), 9.31\left(\mathrm{CH}_{3}\right)$.

\section{Thymyl Butanoate (3d)}

Chromatographic purification gave yellowish oil. $\mathrm{C}_{14} \mathrm{H}_{20} \mathrm{O}_{2}(M=220.31)$; yield 84\%; MS (EI): $\mathrm{m} / \mathrm{z}(\%) 220$ $\left(\mathrm{M}^{+}\right)$(9.7), 151 (6.1), 150 (55.5), 136 (9.5), 135 (100), 115 (5.4), 105 (5.7), 91 (10.4), 71 (9.3), 43 (7.1); RI (HP5-MS): $1544 ;{ }^{1} \mathrm{H}$ NMR (500.13 $\left.\mathrm{MHz} \mathrm{CDCl}_{3}\right): \delta$ (ppm) $7.21(1 \mathrm{H}, \mathrm{d}, J=8 \mathrm{~Hz}, \mathrm{Ar}-\mathrm{H}), 7.04(1 \mathrm{H}, \mathrm{d}, J=8 \mathrm{~Hz}$, Ar-H), 6.82 (1H, s, Ar-H), 2.99 (1H, spt, $J=7 \mathrm{~Hz}, \mathrm{CH})$, $2.58\left(2 \mathrm{H}, \mathrm{t}, J=7 \mathrm{~Hz}, \mathrm{CH}_{2}\right), 2.33\left(3 \mathrm{H}, \mathrm{s}, \mathrm{CH}_{3}\right), 1.84(2 \mathrm{H}$, sxt, $\left.J=7 \mathrm{~Hz}, \mathrm{CH}_{2}\right), 2.1\left(6 \mathrm{H}, \mathrm{d}, J=7 \mathrm{~Hz}, \mathrm{CH}_{3}\right), 1.08(3 \mathrm{H}$, $\left.\mathrm{t}, J=7 \mathrm{~Hz}, \mathrm{CH}_{3}\right) ;{ }^{13} \mathrm{C} \mathrm{NMR}\left(125.76 \mathrm{MHz}, \mathrm{CDCl}_{3}\right): \delta$ (ppm) $172.34(\mathrm{C}=\mathrm{O}), 147.88\left(\mathrm{C}_{\mathrm{Ar}}\right), 136.98\left(\mathrm{C}_{\mathrm{Ar}}\right), 136.49$ $\left(\mathrm{C}_{\mathrm{Ar}}\right), 126.66\left(\mathrm{C}_{\mathrm{Ar}}\right), 125.99\left(\mathrm{C}_{\mathrm{Ar}}\right), 123.24\left(\mathrm{C}_{\mathrm{Ar}}\right), 36.24$ $\left(\mathrm{CH}_{2}\right), 26.90(\mathrm{CH}), 23.07\left(2 \times \mathrm{CH}_{3}\right), 18.55\left(\mathrm{CH}_{2}\right), 13.78$ $\left(\mathrm{CH}_{3}\right)$.

\section{Thymyl 2-Methylpropanoate (3e)}

Chromatographic purification gave colorless oil, $\mathrm{C}_{14} \mathrm{H}_{20} \mathrm{O}_{2}(M=220.31)$; yield 75\%; MS (EI): $\mathrm{m} / z$ (\%) 220 $\left(\mathrm{M}^{+}\right)$(11.4), 150 (59.6), 136 (9.4), 135 (100), 115 (7.3), 105 (8.0), 91 (15.1), 77 (6.8), 71 (18.8), 43 (21.9); RI (HP5-MS): $1495 ;{ }^{1} \mathrm{H}$ NMR (500.13 MHz, $\left.\mathrm{CDCl}_{3}\right): \delta$ (ppm) $7.21(1 \mathrm{H}, \mathrm{d}, J=8 \mathrm{~Hz}, \mathrm{Ar}-\mathrm{H}), 7.03(1 \mathrm{H}, \mathrm{d}, J=7.6$ $\mathrm{Hz}, \mathrm{Ar}-\mathrm{H}), 6.81$ (1H, s, Ar-H), 2.99 (1H, spt, $J=6.9 \mathrm{~Hz}$, $\mathrm{CH}), 2.85$ (1H, spt, $J=7 \mathrm{~Hz}, \mathrm{CH}), 2.33\left(3 \mathrm{H}, \mathrm{s}, \mathrm{CH}_{3}\right) 1.36$ $\left(6 \mathrm{H}, \mathrm{d}, J=7.3 \mathrm{~Hz}, 2 \times \mathrm{CH}_{3}\right), 1.21(6 \mathrm{H}, \mathrm{d}, J=6.9 \mathrm{~Hz}, 2 \times$ $\left.\mathrm{CH}_{3}\right) .{ }^{13} \mathrm{C} \mathrm{NMR}\left(125.76 \mathrm{MHz}, \mathrm{CDCl}_{3}\right): \delta(\mathrm{ppm}) 175.71$ $(\mathrm{C}=\mathrm{O}), 147.99\left(\mathrm{C}_{\mathrm{Ar}}\right), 136.97\left(\mathrm{C}_{\mathrm{Ar}}\right), 136.47\left(\mathrm{C}_{\mathrm{Ar}}\right), 126.62$ $\left(\mathrm{C}_{\mathrm{Ar}}\right), 126.56\left(\mathrm{C}_{\mathrm{Ar}}\right), 122.14\left(\mathrm{C}_{\mathrm{Ar}}\right), 33.95(\mathrm{CH}), 26.78$ $(\mathrm{CH}), 22.99\left(2 \times \mathrm{CH}_{3}\right), 19.20\left(\mathrm{CH}_{3}\right), 19.05\left(2 \times \mathrm{CH}_{3}\right)$.

\section{Thymyl Pentanoate (3f)}

Chromatographic purification gave colorless oil. $\mathrm{C}_{15} \mathrm{H}_{22} \mathrm{O}_{2}(M=234.33)$; yield 87\%; MS (EI): $m / z(\%) 234$ $\left(\mathrm{M}^{+}\right)$(8.1), 151 (7.4), 150 (67.6), 135 (100), 136 (9.3), 115 (5.5), 105 (6.1), 91 (10.7), 85 (8.0), 57 (16.4); RI (HP5-MS): $1640 ;{ }^{1} \mathrm{H}$ NMR (500.13 MHz, $\left.\mathrm{CDCl}_{3}\right): \delta$ (ppm) $7.21(1 \mathrm{H}, \mathrm{d}, J=8 \mathrm{~Hz}, \mathrm{Ar}-\mathrm{H}), 7.04(1 \mathrm{H}, \mathrm{d}, J=8 \mathrm{~Hz}$, Ar-H), 6.82 (1H, s, Ar-H), 2.99 (1H, spt, $J=14 \mathrm{~Hz}, \mathrm{CH})$, $2.6\left(2 \mathrm{H}, \mathrm{t}, J=7.6 \mathrm{~Hz}, \mathrm{CH}_{2}\right), 2.33\left(3 \mathrm{H}, \mathrm{s}, \mathrm{CH}_{3}\right), 1.79(2 \mathrm{H}$, quin, $\left.J=7.5 \mathrm{~Hz}, \mathrm{CH}_{2}\right), 1.49\left(2 \mathrm{H}\right.$, sxt, $\left.J=15 \mathrm{~Hz}, \mathrm{CH}_{2}\right)$, $1.21\left(6 \mathrm{H}, \mathrm{d}, J=6.9 \mathrm{~Hz}, 2 \times \mathrm{CH}_{3}\right), 1.01(3 \mathrm{H}, \mathrm{t}, J=7.5 \mathrm{~Hz}$, $\left.\mathrm{CH}_{3}\right) ;{ }^{13} \mathrm{C}$ NMR (125.76 MHz, $\left.\mathrm{CDCl}_{3}\right): \delta(\mathrm{ppm}) 172.45$ $(\mathrm{C}=\mathrm{O}), 147.90\left(\mathrm{C}_{\mathrm{Ar}}\right), 136.95\left(\mathrm{C}_{\mathrm{Ar}}\right), 136.45\left(\mathrm{C}_{\mathrm{Ar}}\right), 126.96$ $\left(\mathrm{C}_{\mathrm{Ar}}\right), 126.3\left(\mathrm{C}_{\mathrm{Ar}}\right), 122.7\left(\mathrm{C}_{\mathrm{Ar}}\right), 34.06\left(\mathrm{CH}_{2}\right), 27.06\left(\mathrm{CH}_{2}\right)$, $27.02(\mathrm{CH}), 22.28\left(\mathrm{CH}_{2}\right), 22.96\left(2 \times \mathrm{CH}_{3}\right), 20.76\left(\mathrm{CH}_{3}-\right.$ Ar), $18.55\left(\mathrm{CH}_{2}\right), 13.8\left(\mathrm{CH}_{3}\right)$.

\section{Thymyl 3-Methylbutanoate (3g)}

Chromatographic purification gave colorless oil. $\mathrm{C}_{15} \mathrm{H}_{22} \mathrm{O}_{2}(M=234.33)$; yield 81\%; MS (EI): $\mathrm{m} / z$ (\%) 234 $\left(\mathrm{M}^{+}\right)$(8.9), 151 (8.3), 150 (71.4), 135 (100), 136 (9.3), 115 (5.4), 105 (6.8), 91 (11.0), 85 (9.3), 52 (21.5); RI (HP5-MS): $1590 ;{ }^{1} \mathrm{H}$ NMR $\left(500.13 \mathrm{MHz} \mathrm{CDCl}_{3}\right): \delta$ (ppm) $7.21(1 \mathrm{H}, \mathrm{d}, J=8 \mathrm{~Hz}, \mathrm{Ar}-\mathrm{H}), 7.04(1 \mathrm{H}, \mathrm{d}, J=7.6$ $\mathrm{Hz}, \mathrm{Ar}-\mathrm{H}), 6.81$ (1H, s, Ar-H), $3.00(1 \mathrm{H}, \mathrm{spt}, J=6.9 \mathrm{~Hz}$, $\mathrm{CH}), 2.48\left(2 \mathrm{H}, \mathrm{d}, J=6.9 \mathrm{~Hz}, \mathrm{CH}_{2}\right), 2.33\left(3 \mathrm{H}, \mathrm{s}, \mathrm{CH}_{3}\right)$, $2.28(1 \mathrm{H}, \mathrm{m}, \mathrm{CH}), 1.49\left(2 \mathrm{H}, \mathrm{sxt}, J=15 \mathrm{~Hz}, \mathrm{CH}_{2}\right), 1.20$ $\left(6 \mathrm{H}, \mathrm{d}, J=6.9 \mathrm{~Hz}, 2 \times \mathrm{CH}_{3}\right), 1.09(6 \mathrm{H}, \mathrm{d}, J=6.6 \mathrm{~Hz}, 2 \times$ $\left.\mathrm{CH}_{3}\right) ;{ }^{13} \mathrm{C}$ NMR $\left(125.76 \mathrm{MHz}, \mathrm{CDCl}_{3}\right): \delta(\mathrm{ppm}) 171.74$ $(\mathrm{C}=\mathrm{O}), 147.87\left(\mathrm{C}_{\mathrm{Ar}}\right), 136.99\left(\mathrm{C}_{\mathrm{Ar}}\right), 136.45\left(\mathrm{C}_{\mathrm{Ar}}\right), 126.99$ $\left(\mathrm{C}_{\mathrm{Ar}}\right), 126.32\left(\mathrm{C}_{\mathrm{Ar}}\right), 122.70\left(\mathrm{C}_{\mathrm{Ar}}\right), 43.34\left(\mathrm{CH}_{2}\right), 26.98$ 
$\left(\mathrm{CH}_{2}\right), 25.98(\mathrm{CH}), 23.01\left(2 \times \mathrm{CH}_{3}\right), 22.44\left(2 \times \mathrm{CH}_{3}\right)$, $20,78\left(\mathrm{CH}_{3}-\mathrm{Ar}\right)$.

\section{Thymyl Hexanoate (3h)}

Chromatographic purification gave colorless oil, $\mathrm{C}_{16} \mathrm{H}_{24} \mathrm{O}_{2}(M=248.36)$; yield 79\%; MS (EI): $\mathrm{m} / z$ (\%) 248 $\left(\mathrm{M}^{+}\right)$(7.3), 151 (8.9), 150 (79.2), 136 (9.4), 135 (100), 105 (6.7), 99 (6.6), 91 (10.5), 71 (9.7), 43 (9.3); RI (HP5-MS): 1738; ${ }^{1} \mathrm{H}$ NMR $\left(500.13 \mathrm{MHz}, \mathrm{CDCl}_{3}\right): \delta(\mathrm{ppm}) 7.20$ $(1 \mathrm{H}, \mathrm{d}, J=8 \mathrm{~Hz}, \mathrm{Ar}-\mathrm{H}), 7.03(1 \mathrm{H}, \mathrm{d}, J=7.6 \mathrm{~Hz}, \mathrm{Ar}-\mathrm{H})$, $6.81(1 \mathrm{H}, \mathrm{s}, \mathrm{Ar}-\mathrm{H}), 2.98(1 \mathrm{H}, \mathrm{spt}, J=6.9 \mathrm{~Hz}, \mathrm{CH}), 2.58$ $\left(2 \mathrm{H}, \mathrm{t}, J=7.6 \mathrm{~Hz}, \mathrm{CH}_{2}\right), 2.32\left(3 \mathrm{H}, \mathrm{s}, \mathrm{CH}_{3}\right), 1.79(2 \mathrm{H}$, quin, $\left.J=7.5 \mathrm{~Hz}, \mathrm{CH}_{2}\right), 1.41\left(4 \mathrm{H}, \mathrm{m}, \mathrm{CH}_{2}\right), 1.20(6 \mathrm{H}, \mathrm{d}, J=6.9$ $\left.\mathrm{Hz}, \mathrm{CH}_{3}\right), 0.95\left(3 \mathrm{H}, \mathrm{m}, \mathrm{CH}_{3}\right) ;{ }^{13} \mathrm{C}$ NMR $(125.76 \mathrm{MHz}$, $\left.\mathrm{CDCl}_{3}\right): \delta(\mathrm{ppm}) 172.50(\mathrm{C}=\mathrm{O}), 147.90\left(\mathrm{C}_{\mathrm{Ar}}\right), 137.00$ $\left(\mathrm{C}_{\mathrm{Ar}}\right), 136.50\left(\mathrm{C}_{\mathrm{Ar}}\right), 127.00\left(\mathrm{C}_{\mathrm{Ar}}\right), 126.3\left(\mathrm{C}_{\mathrm{Ar}}\right), 122.7\left(\mathrm{C}_{\mathrm{Ar}}\right)$, $34.38\left(\mathrm{CH}_{2}\right), 31.36\left(\mathrm{CH}_{2}\right), 27.08(\mathrm{CH}), 24.76\left(\mathrm{CH}_{2}\right), 23.03$ $\left(2 \times \mathrm{CH}_{3}\right), 22.36\left(\mathrm{CH}_{2}\right), 20.83\left(\mathrm{CH}_{3}\right), 13.94\left(\mathrm{CH}_{3}\right)$.

\section{Thymyl Heptanoate (3i)}

Chromatographic purification gave colorless oil. $\mathrm{C}_{17} \mathrm{H}_{26} \mathrm{O}_{2}(M=262.39)$; yield 84\%; MS (EI): $\mathrm{m} / z$ (\%) 262 $\left(\mathrm{M}^{+}\right)$(7.6), 151 (10.8), 150 (95.6), 149 (5.3), 136 (9.4), 135 (100), 113 (5.6), 105 (6.4), 91 (9.8), 43 (9.4); RI (HP5-MS): $1840 ;{ }^{1} \mathrm{H}$ NMR $\left(500.13 \mathrm{MHz}, \mathrm{CDCl}_{3}\right): \delta$ (ppm) $7.21(1 \mathrm{H}, \mathrm{d}, J=8 \mathrm{~Hz}, \mathrm{Ar}-\mathrm{H}), 7.03(1 \mathrm{H}, \mathrm{d}, J=8 \mathrm{~Hz}$, Ar-H), $6.81(1 \mathrm{H}, \mathrm{s}, \mathrm{Ar}-\mathrm{H}), 2.99(1 \mathrm{H}, \mathrm{spt}, J=14 \mathrm{~Hz}, \mathrm{CH})$, $2.59\left(2 \mathrm{H}, \mathrm{t}, J=8 \mathrm{~Hz}, \mathrm{CH}_{2}\right), 2.33\left(3 \mathrm{H}, \mathrm{s}, \mathrm{CH}_{3}\right), 1.79(2 \mathrm{H}$, $\left.\mathrm{m}, \mathrm{CH}_{2}\right), 1.45\left(2 \mathrm{H}, \mathrm{m}, \mathrm{CH}_{2}\right), 1.36\left(4 \mathrm{H}, \mathrm{m}, \mathrm{CH}_{2}\right), 1.22(6 \mathrm{H}$, $\left.\mathrm{m}, \mathrm{CH}_{3}\right), 0.92\left(3 \mathrm{H}, \mathrm{m}, \mathrm{CH}_{3}\right) ;{ }^{13} \mathrm{C}$ NMR $(125.76 \mathrm{MHz}$, $\left.\mathrm{CDCl}_{3}\right): \delta(\mathrm{ppm}) 172.53(\mathrm{C}=\mathrm{O}), 147.91\left(\mathrm{C}_{\mathrm{Ar}}\right), 136.99$ $\left(\mathrm{C}_{\mathrm{Ar}}\right), 136.49\left(\mathrm{C}_{\mathrm{Ar}}\right), 126.99\left(\mathrm{C}_{\mathrm{Ar}}\right), 126.33\left(\mathrm{C}_{\mathrm{Ar}}\right), 122.73$ $\left(\mathrm{C}_{\mathrm{Ar}}\right), 34.39\left(\mathrm{CH}_{2}\right), 31.44\left(\mathrm{CH}_{2}\right), 28.84\left(\mathrm{CH}_{2}\right), 27.03$ (CH), $25.0\left(\mathrm{CH}_{2}\right), 23.0\left(2 \times \mathrm{CH}_{3}\right), 22.49\left(\mathrm{CH}_{2}\right), 20.8$ $\left(\mathrm{CH}_{3}-\mathrm{Ar}\right), 14.01\left(\mathrm{CH}_{3}\right)$.

\section{Thymyl Octanoate (3j)}

Chromatographic purification gave colorless oil. $\mathrm{C}_{18} \mathrm{H}_{28} \mathrm{O}_{2}(M=276.41)$; yield 85\%; MS (EI): $\mathrm{m} / z$ (\%) 276 $\left(\mathrm{M}^{+}\right) 151$ (11.3), 150 (100), 136 (9.3), 135 (98.3), 109 (6.4), 105 (7.2), 91 (10.9), 57 (27.2), 55 (9.4), 43 (6.3); RI (HP5-MS): 1938; ${ }^{1} \mathrm{H}$ NMR (500.13 MHz, $\left.\mathrm{CDCl}_{3}\right)$ : $\delta$ (ppm) $7.21(1 \mathrm{H}, \mathrm{d}, J=7.9 \mathrm{~Hz}, \mathrm{Ar}-\mathrm{H}), 7.03(1 \mathrm{H}, \mathrm{d}, J=7.9$ $\mathrm{Hz}, \mathrm{Ar}-\mathrm{H}), 6.81(1 \mathrm{H}, \mathrm{s}, \mathrm{Ar}-\mathrm{H}), 2.99(1 \mathrm{H}, \mathrm{spt}, J=6.9 \mathrm{~Hz}$, $\mathrm{CH}), 2.59\left(2 \mathrm{H}, \mathrm{t}, J=7.5 \mathrm{~Hz}, \mathrm{CH}_{2}\right), 2.33\left(3 \mathrm{H}, \mathrm{s}, \mathrm{CH}_{3}\right), 1.79$ $\left(2 \mathrm{H}\right.$, quin, $\left.J=7.5 \mathrm{~Hz}, \mathrm{CH}_{2}\right), 1.47-1.29\left(8 \mathrm{H}, \mathrm{m}, \mathrm{CH}_{2}\right), 1.21$ $\left(6 \mathrm{H}, \mathrm{bd}, \mathrm{CH}_{3}\right), 0.92\left(3 \mathrm{H}, \mathrm{t}, J=6.8 \mathrm{~Hz}, \mathrm{CH}_{3}\right) ;{ }^{13} \mathrm{C} \mathrm{NMR}$ $\left(125.76 \mathrm{MHz}, \mathrm{CDCl}_{3}\right): \delta(\mathrm{ppm}) 174.20(\mathrm{C}=\mathrm{O}), 148.00$ $\left(\mathrm{C}_{\mathrm{Ar}}\right), 136.80\left(\mathrm{C}_{\mathrm{Ar}}\right), 136.09\left(\mathrm{C}_{\mathrm{Ar}}\right), 127.00\left(\mathrm{C}_{\mathrm{Ar}}\right), 126.30$ $\left(\mathrm{C}_{\mathrm{Ar}}\right), 122.80\left(\mathrm{C}_{\mathrm{Ar}}\right), 36.10\left(\mathrm{CH}_{2}\right), 34.00\left(\mathrm{CH}_{2}\right), 32.04$ $\left(\mathrm{CH}_{2}\right), 29.00\left(\mathrm{CH}_{2}\right), 28.00(\mathrm{CH}), 26.00\left(\mathrm{CH}_{2}\right), 23.0(2 \times$ $\left.\mathrm{CH}_{3}\right), 22.10\left(\mathrm{CH}_{2}\right), 20.5\left(\mathrm{CH}_{3}-\mathrm{Ar}\right), 14.00\left(\mathrm{CH}_{3}\right)$.

\section{Thymyl Nonanoate (3k)}

Chromatographic purification gave colorless oil. $\mathrm{C}_{19} \mathrm{H}_{30} \mathrm{O}_{2}(M=290.44)$; yield 79.80\%; MS (EI): $\mathrm{m} / \mathrm{z}(\%)$
$290\left(\mathrm{M}^{+}\right)$(5.7), 151 (12.0), 150 (100), 141 (3.2), 136 (7.7), 135 (79.0), 121 (3.4), 117 (2.0), 115 (2.3), 91 (3.3); RI (HP5-MS): 2044; ${ }^{1} \mathrm{H}$ NMR $\left(500.13 \mathrm{MHz}, \mathrm{CDCl}_{3}\right)$ : $\delta$ (ppm) 7.24-7.19 (1H, m, Ar-H), 7.06-7.01 (1H, bd, Ar$\mathrm{H}), 6.84-6.79(1 \mathrm{H}, \mathrm{bs}, \mathrm{Ar}-\mathrm{H}), 3.04-2.93(1 \mathrm{H}, \mathrm{bm}, \mathrm{CH})$, 2.63-2.56 (2H, bm, $\left.\mathrm{CH}_{2}\right), 2.36-2.31\left(3 \mathrm{H}, \mathrm{s}, \mathrm{CH}_{3}\right)$, 1.84-1.75 (2H, bm, $\left.\mathrm{CH}_{2}\right), 1.49-1.26\left(10 \mathrm{H}, \mathrm{bm}, \mathrm{CH}_{2}\right)$, 1.24-1.18 $\left(6 \mathrm{H}, \mathrm{bt}, \mathrm{CH}_{3}\right), 0.95-0.87\left(3 \mathrm{H}, \mathrm{bm}, \mathrm{CH}_{3}\right) ;{ }^{13} \mathrm{C}$ NMR (125.76 MHz, $\left.\mathrm{CDCl}_{3}\right): \delta(\mathrm{ppm}) 172.53(\mathrm{C}=\mathrm{O})$, $147.91\left(\mathrm{C}_{\mathrm{Ar}}\right), 136.99\left(\mathrm{C}_{\mathrm{Ar}}\right), 136.49\left(\mathrm{C}_{\mathrm{Ar}}\right), 126.99\left(\mathrm{C}_{\mathrm{Ar}}\right)$, $126.33\left(\mathrm{C}_{\mathrm{Ar}}\right), 122.73\left(\mathrm{C}_{\mathrm{Ar}}\right), 36.10\left(\mathrm{CH}_{2}\right), 34.20\left(\mathrm{CH}_{2}\right)$, $32.00\left(\mathrm{CH}_{2}\right), 28.80\left(2 \times \mathrm{CH}_{2}\right), 27.60(\mathrm{CH}), 25.60\left(\mathrm{CH}_{2}\right)$, $23.0\left(2 \times \mathrm{CH}_{3}\right), 22.50\left(\mathrm{CH}_{2}\right), 20.8\left(\mathrm{CH}_{3}-\mathrm{Ar}\right), 14.01\left(\mathrm{CH}_{3}\right)$.

\section{Thymyl Decanoate (3I)}

Chromatographic purification gave colorless oil. $\mathrm{C}_{20} \mathrm{H}_{32} \mathrm{O}_{2}(M=304.47)$; yield $75.50 \%$; $\mathrm{MS}(\mathrm{EI}): \mathrm{m} / \mathrm{z}(\%)$ : $304\left(\mathrm{M}^{+}\right)$(4.6), 151 (12.3), 150 (100), 136 (6.7), 135 (71.9), 109 (5.3), 91 (5.7), 71 (4.8), 57 (4.8), 55 (5.5); RI (HP5-MS): 2147; ${ }^{1} \mathrm{H}$ NMR $\left(500.13 \mathrm{MHz}, \mathrm{CDCl}_{3}\right): \delta$ (ppm) $7.21(1 \mathrm{H}, \mathrm{d}, J=8 \mathrm{~Hz}, \mathrm{Ar}-\mathrm{H}), 7.03(1 \mathrm{H}, \mathrm{d}, J=7.6$ $\mathrm{Hz}, \mathrm{Ar}-\mathrm{H}), 6.82(1 \mathrm{H}, \mathrm{s}, \mathrm{Ar}-\mathrm{H}), 2.99(1 \mathrm{H}, \mathrm{spt}, J=6.9 \mathrm{~Hz}$, $\mathrm{CH}), 2.59\left(2 \mathrm{H}, \mathrm{t}, J=7.5 \mathrm{~Hz}, \mathrm{CH}_{2}\right), 2.33\left(3 \mathrm{H}, \mathrm{s}, \mathrm{CH}_{3}\right), 1.8$ (2H, quin, $\left.J=7.5 \mathrm{~Hz}, \mathrm{CH}_{2}\right), 1.49-1.25\left(12 \mathrm{H}, \mathrm{bm}, \mathrm{CH}_{2}\right)$, $1.21\left(6 \mathrm{H}, \mathrm{d}, J=6.9 \mathrm{~Hz}, \mathrm{CH}_{3}\right), 0.91(3 \mathrm{H}, \mathrm{t}, J=6.2 \mathrm{~Hz}$, $\left.\mathrm{CH}_{3}\right) ;{ }^{13} \mathrm{C}$ NMR $\left(125.76 \mathrm{MHz}, \mathrm{CDCl}_{3}\right): \delta(\mathrm{ppm}) 172.52$ $(\mathrm{C}=\mathrm{O}), 147.91\left(\mathrm{C}_{\mathrm{Ar}}\right), 136.99\left(\mathrm{C}_{\mathrm{Ar}}\right), 136.48\left(\mathrm{C}_{\mathrm{Ar}}\right), 126.99$ $\left(\mathrm{C}_{\mathrm{Ar}}\right), 126.33\left(\mathrm{C}_{\mathrm{Ar}}\right), 122.73\left(\mathrm{C}_{\mathrm{Ar}}\right), 34.39\left(\mathrm{CH}_{2}\right), 31.85$ $\left(\mathrm{CH}_{2}\right), 29.43\left(\mathrm{CH}_{2}\right), 29.25\left(2 \times \mathrm{CH}_{2}\right), 29.17\left(\mathrm{CH}_{2}\right), 27.04$ $(\mathrm{CH}), 25.05\left(\mathrm{CH}_{2}\right), 23.0\left(2 \times \mathrm{CH}_{3}\right), 22.67\left(\mathrm{CH}_{2}\right), 20.8$ $\left(\mathrm{CH}_{3}-\mathrm{Ar}\right), 14.09\left(\mathrm{CH}_{3}\right)$.

\section{Thymyl Undecanoate (3m)}

Chromatographic purification gave colorless oil. $\mathrm{C}_{21} \mathrm{H}_{34} \mathrm{O}_{2}(M=318.49)$; yield $82.00 \%$; MS (EI): $\mathrm{m} / z(\%)$ $318\left(\mathrm{M}^{+}\right) 151$ (7.9), 150 (100), 149 (7.4), 135 (80.1), 134 (6.8), 105 (7.0), 91 (13.3), 57 (18.4), 55 (20.2), 43 (12.1); RI (HP5-MS): 2245; ${ }^{1} \mathrm{H}$ NMR (500.13 MHz, $\mathrm{CDCl}_{3}$ ): $\delta$ (ppm) $7.21(1 \mathrm{H}, \mathrm{d}, J=8 \mathrm{~Hz}, \mathrm{Ar}-\mathrm{H}), 7.03(1 \mathrm{H}, \mathrm{d}, J=7.6$ $\mathrm{Hz}, \mathrm{Ar}-\mathrm{H}), 6.82(1 \mathrm{H}, \mathrm{s}, \mathrm{Ar}-\mathrm{H}), 2.99(1 \mathrm{H}, \mathrm{spt}, J=13.9 \mathrm{~Hz}$, $\mathrm{CH}), 2.59\left(2 \mathrm{H}, \mathrm{t}, J=7.46 \mathrm{~Hz}, \mathrm{CH}_{2}\right), 2.33\left(3 \mathrm{H}, \mathrm{s}, \mathrm{CH}_{3}\right)$, $1.79\left(2 \mathrm{H}\right.$, quin, $\left.J=7.54 \mathrm{~Hz}, \mathrm{CH}_{2}\right), 1.41-1.48(2 \mathrm{H}, \mathrm{m}$, $\left.\mathrm{CH}_{2}\right), 1.26-1.39\left(12 \mathrm{H}, \mathrm{m}, \mathrm{CH}_{2}\right), 1.21(6 \mathrm{H}, \mathrm{d}, J=6.94 \mathrm{~Hz}$, $\left.\mathrm{CH}_{3}\right), 0.91\left(3 \mathrm{H}, \mathrm{t}, J=6.76 \mathrm{~Hz}, \mathrm{CH}_{3}\right) ;{ }^{13} \mathrm{C}$ NMR $(125.76$ $\left.\mathrm{MHz}, \mathrm{CDCl}_{3}\right): \delta(\mathrm{ppm}) 172.52(\mathrm{C}=\mathrm{O}), 147.91\left(\mathrm{C}_{\mathrm{Ar}}\right)$, $136.99\left(\mathrm{C}_{\mathrm{Ar}}\right), 136.48\left(\mathrm{C}_{\mathrm{Ar}}\right), 126.99\left(\mathrm{C}_{\mathrm{Ar}}\right), 126.33\left(\mathrm{C}_{\mathrm{Ar}}\right)$, $122.73\left(\mathrm{C}_{\mathrm{Ar}}\right), 34.39\left(\mathrm{CH}_{2}\right), 31.88\left(\mathrm{CH}_{2}\right), 29.54\left(\mathrm{CH}_{2}\right)$, $29.47\left(\mathrm{CH}_{2}\right), 29.30\left(\mathrm{CH}_{2}\right), 29.26\left(\mathrm{CH}_{2}\right), 29.17\left(\mathrm{CH}_{2}\right)$, $27.04(\mathrm{CH}), 25.05\left(\mathrm{CH}_{2}\right), 23.0\left(2 \times \mathrm{CH}_{3}\right), 22.67\left(\mathrm{CH}_{2}\right)$, $20.8\left(\mathrm{CH}_{3}-\mathrm{Ar}\right), 14.10\left(\mathrm{CH}_{3}\right)$.

\section{Thymyl Dodecanoate (3n)}

Chromatographic purification gave colorless oil. $\mathrm{C}_{22} \mathrm{H}_{36} \mathrm{O}_{2}(M=332.52)$; yield $76.30 \%$; MS (EI): $\mathrm{m} / \mathrm{z}(\%)$ $332\left(\mathrm{M}^{+}\right)(4.4), 151(15.2), 150$ (100), $136(6.7), 135$ (67.8), 109 (7.3), 91 (4.6), 57 (6.4), 55 (5.6), 43 (3.6); RI 
(HP5-MS): $2355 ;{ }^{1} \mathrm{H}$ NMR $\left(500.13 \mathrm{MHz}, \mathrm{CDCl}_{3}\right): \delta$ (ppm) $7.21(1 \mathrm{H}, \mathrm{d}, J=8 \mathrm{~Hz}, \mathrm{Ar}-\mathrm{H}), 7.03(1 \mathrm{H}, \mathrm{d}, J=8 \mathrm{~Hz}$, Ar-H), $6.81(1 \mathrm{H}, \mathrm{s}, \mathrm{Ar}-\mathrm{H}), 2.98(1 \mathrm{H}, \mathrm{spt}, J=6.9 \mathrm{~Hz}, \mathrm{CH})$, $2.59\left(2 \mathrm{H}, \mathrm{t}, J=7.6 \mathrm{~Hz}, \mathrm{CH}_{2}\right), 2.33\left(3 \mathrm{H}, \mathrm{s}, \mathrm{CH}_{3}\right), 1.79(2 \mathrm{H}$, quin, $\left.J=7.5 \mathrm{~Hz}, \mathrm{CH}_{2}\right), 1.48-1.40\left(14 \mathrm{H}, \mathrm{m}, \mathrm{CH}_{2}\right), 1.20$ $\left(6 \mathrm{H}, \mathrm{d}, J=6.9 \mathrm{~Hz}, \mathrm{CH}_{3}\right), 0.90\left(3 \mathrm{H}, \mathrm{t}, J=6.8 \mathrm{~Hz}, \mathrm{CH}_{3}\right)$; ${ }^{13} \mathrm{C}$ NMR $\left(125.76 \mathrm{MHz}, \mathrm{CDCl}_{3}\right): \delta(\mathrm{ppm}) 172.55(\mathrm{C}=\mathrm{O})$, $147.91\left(\mathrm{C}_{\mathrm{Ar}}\right), 136.99\left(\mathrm{C}_{\mathrm{Ar}}\right), 136.49\left(\mathrm{C}_{\mathrm{Ar}}\right), 126.99\left(\mathrm{C}_{\mathrm{Ar}}\right)$, $126.33\left(\mathrm{C}_{\mathrm{Ar}}\right), 122.73\left(\mathrm{C}_{\mathrm{Ar}}\right), 34.40\left(\mathrm{CH}_{2}\right), 31.90\left(\mathrm{CH}_{2}\right)$, $29.60\left(2 \times \mathrm{CH}_{2}\right), 29.47\left(\mathrm{CH}_{2}\right), 29.33\left(\mathrm{CH}_{2}\right), 29.18\left(\mathrm{CH}_{2}\right)$, $29.17\left(\mathrm{CH}_{2}\right), 27.04(\mathrm{CH}), 25.06\left(\mathrm{CH}_{2}\right), 23.01\left(2 \times \mathrm{CH}_{3}\right)$, $22.68\left(\mathrm{CH}_{2}\right), 20.81\left(\mathrm{CH}_{3}-\mathrm{Ar}\right), 14.10\left(\mathrm{CH}_{3}\right)$.

\section{Thymyl Tridecanoate (3o)}

Chromatographic purification gave colorless oil, $\mathrm{C}_{23} \mathrm{H}_{38} \mathrm{O}_{2}(M=346.55)$; yield $85.1 \%$; MS (EI): $\mathrm{m} / z(\%)$ $346\left(\mathrm{M}^{+}\right)$(2.4), $347(0.6), 197(0.9), 152(0.9), 151$ (12.9), 150 (100), 137 (0.4), 136 (5.0), 135 (54.2), 119 (0.5); RI (HP5-MS): 2453; ${ }^{1} \mathrm{H}$ NMR (500.13 MHz, $\mathrm{CDCl}_{3}$ ): $\delta$ (ppm) $7.21(1 \mathrm{H}, \mathrm{d}, J=7.98 \mathrm{~Hz}, \mathrm{Ar}-\mathrm{H}), 7.03(1 \mathrm{H}, \mathrm{d}, J=$ $7.98 \mathrm{~Hz}, \mathrm{Ar}-\mathrm{H}), 6.81(1 \mathrm{H}, \mathrm{s}, \mathrm{Ar}-\mathrm{H}), 2.98(1 \mathrm{H}, \mathrm{spt}, J=13.9$ $\mathrm{Hz}, \mathrm{CH}), 2.59\left(2 \mathrm{H}, \mathrm{t}, J=7.63 \mathrm{~Hz}, \mathrm{CH}_{2}\right), 2.33(3 \mathrm{H}, \mathrm{s}$, $\left.\mathrm{CH}_{3}\right), 1.79\left(2 \mathrm{H}\right.$, quin, $\left.J=7.54 \mathrm{~Hz}, \mathrm{CH}_{2}\right), 1.26-1.48(16 \mathrm{H}$, m, $\left.\mathrm{CH}_{2}\right), 1.20\left(6 \mathrm{H}, \mathrm{d}, J=6.94 \mathrm{~Hz}, \mathrm{CH}_{3}\right), 0.90(3 \mathrm{H}, \mathrm{t}, J=$ $\left.6.76 \mathrm{~Hz}, \mathrm{CH}_{3}\right) ;{ }^{13} \mathrm{C} \mathrm{NMR}\left(125.76 \mathrm{MHz}, \mathrm{CDCl}_{3}\right): \delta(\mathrm{ppm})$ $172.5(\mathrm{C}=\mathrm{O}), 147.9\left(\mathrm{C}_{\mathrm{Ar}}\right), 137\left(\mathrm{C}_{\mathrm{Ar}}\right), 136.5\left(\mathrm{C}_{\mathrm{Ar}}\right), 127.0$ $\left(\mathrm{C}_{\mathrm{Ar}}\right), 126.3\left(\mathrm{C}_{\mathrm{Ar}}\right), 122.7\left(\mathrm{C}_{\mathrm{Ar}}\right), 34.43\left(\mathrm{CH}_{2}\right), 31.94\left(\mathrm{CH}_{2}\right)$, $29.67\left(2 \times \mathrm{CH}_{2}\right), 29.51\left(\mathrm{CH}_{2}\right), 29.38\left(\mathrm{CH}_{2}\right), 29.3\left(\mathrm{CH}_{2}\right)$, $29.22\left(\mathrm{CH}_{2}\right), 27.08(\mathrm{CH}), 25.09\left(\mathrm{CH}_{2}\right), 23.04\left(2 \times \mathrm{CH}_{3}\right)$, $22.71\left(\mathrm{CH}_{2}\right), 20.83\left(\mathrm{CH}_{3}-\mathrm{Ar}\right), 14.13\left(\mathrm{CH}_{3}\right)$.

\section{Thymyl Tetradecanoate (3p)}

Chromatographic purification gave colorless oil. $\mathrm{C}_{24} \mathrm{H}_{40} \mathrm{O}_{2}(M=360.57)$; yield $72.05 \%$; MS (EI): $\mathrm{m} / \mathrm{z}(\%)$ $360\left(\mathrm{M}^{+}\right)(1.9), 152(0.9), 151$ (12.7), 150 (100), 135 (50.7), 134 (4.8), 121 (1.3), 117 (0.7), 115 (1.9), 109 (5.1); RI (HP5-MS): 2555; ${ }^{1} \mathrm{H}$ NMR (500.13 MHz, CDC$\left.1_{3}\right): \delta(\mathrm{ppm}) 7.21(1 \mathrm{H}, \mathrm{d}, J=8 \mathrm{~Hz}, \mathrm{Ar}-\mathrm{H}), 7.03(1 \mathrm{H}, \mathrm{d}, J=$ $7.6 \mathrm{~Hz}, \mathrm{Ar}-\mathrm{H}), 6.81(1 \mathrm{H}, \mathrm{s}, \mathrm{Ar}-\mathrm{H}), 2.99(1 \mathrm{H}, \mathrm{spt}, J=6.9$ $\mathrm{Hz}, \mathrm{CH}), 2.59\left(2 \mathrm{H}, \mathrm{t}, J=7.6 \mathrm{~Hz}, \mathrm{CH}_{2}\right), 2.33\left(3 \mathrm{H}, \mathrm{s}, \mathrm{CH}_{3}\right)$, $1.79\left(2 \mathrm{H}\right.$, quin, $\left.J=7.5 \mathrm{~Hz}, \mathrm{CH}_{2}\right), 1.49-1.25(20 \mathrm{H}, \mathrm{m}$, $\left.\mathrm{CH}_{2}\right), 1.21\left(6 \mathrm{H}, \mathrm{d}, J=6.9 \mathrm{~Hz}, \mathrm{CH}_{3}\right), 0.91(3 \mathrm{H}, \mathrm{t}, J=6.9$ $\left.\mathrm{Hz}, \mathrm{CH}_{3}\right) ;{ }^{13} \mathrm{C}$ NMR $\left(125.76 \mathrm{MHz}, \mathrm{CDCl}_{3}\right): \delta(\mathrm{ppm})$ $172.53(\mathrm{C}=\mathrm{O}), 147.93\left(\mathrm{C}_{\mathrm{Ar}}\right), 136.99\left(\mathrm{C}_{\mathrm{Ar}}\right), 136.48\left(\mathrm{C}_{\mathrm{Ar}}\right)$, $126.99\left(\mathrm{C}_{\mathrm{Ar}}\right), 126.33\left(\mathrm{C}_{\mathrm{Ar}}\right), 122.73\left(\mathrm{C}_{\mathrm{Ar}}\right), 34.39\left(\mathrm{CH}_{2}\right)$, $31.92\left(\mathrm{CH}_{2}\right), 29.67\left(\mathrm{CH}_{2}\right), 29.65\left(2 \times \mathrm{CH}_{2}\right), 29.60\left(\mathrm{CH}_{2}\right)$, $29.48\left(\mathrm{CH}_{2}\right), 29.35\left(\mathrm{CH}_{2}\right), 29.27\left(\mathrm{CH}_{2}\right), 29.19\left(\mathrm{CH}_{2}\right)$, $27.04(\mathrm{CH}), 25.06\left(\mathrm{CH}_{2}\right), 23.00\left(2 \times \mathrm{CH}_{3}\right), 22.68\left(\mathrm{CH}_{2}\right)$, $20.80\left(\mathrm{CH}_{3}-\mathrm{Ar}\right), 14.10\left(\mathrm{CH}_{3}\right)$.

\section{Thymyl Pentadecanoate (3q)}

Chromatographic purification gave colorless oil. $\mathrm{C}_{25} \mathrm{H}_{42} \mathrm{O}_{2}(M=374.60)$; yield $68.90 \%$; MS (EI): $m / z(\%)$ $374\left(\mathrm{M}^{+}\right)$(5.3), 151 (23.9), 150 (100), 149 (7.5), 136 (8.3), 135 (76.4), 109 (11.9), 71 (4.4), 57 (7.0), 55 (6.2); RI (HP5-MS): 2669; ${ }^{1} \mathrm{H}$ NMR (500.13 MHz, $\left.\mathrm{CDCl}_{3}\right): \delta$ (ppm) $7.21(1 \mathrm{H}, \mathrm{d}, J=8 \mathrm{~Hz}, \mathrm{Ar}-\mathrm{H}), 7.03(1 \mathrm{H}, \mathrm{d}, J=8 \mathrm{~Hz}$, Ar-H), $6.81(1 \mathrm{H}, \mathrm{s}, \mathrm{Ar}-\mathrm{H}), 2.99(1 \mathrm{H}, \mathrm{spt}, J=6.90 \mathrm{~Hz}$, $\mathrm{CH}), 2.59\left(2 \mathrm{H}, \mathrm{t}, J=7.6, \mathrm{CH}_{2}\right), 2.33\left(3 \mathrm{H}, \mathrm{s}, \mathrm{CH}_{3}\right), 1.79$ $\left(2 \mathrm{H}\right.$, quin, $\left.J=7.5 \mathrm{~Hz}, \mathrm{CH}_{2}\right), 1.48-1.24\left(22 \mathrm{H}, \mathrm{bm}, \mathrm{CH}_{2}\right)$, $1.21\left(6 \mathrm{H}, \mathrm{d}, J=6.90 \mathrm{~Hz}, \mathrm{CH}_{3}\right), 0.9(3 \mathrm{H}, \mathrm{t}, J=6.90 \mathrm{~Hz}$, $\left.\mathrm{CH}_{3}\right) ;{ }^{13} \mathrm{C}$ NMR $\left(125.76 \mathrm{MHz}, \mathrm{CDCl}_{3}\right): \delta(\mathrm{ppm}) 172.54$ $(\mathrm{C}=\mathrm{O}), 147.92\left(\mathrm{C}_{\mathrm{Ar}}\right), 136.99\left(\mathrm{C}_{\mathrm{Ar}}\right), 136.49\left(\mathrm{C}_{\mathrm{Ar}}\right), 126.99$ $\left(\mathrm{C}_{\mathrm{Ar}}\right), 126.33\left(\mathrm{C}_{\mathrm{Ar}}\right), 122.73\left(\mathrm{C}_{\mathrm{Ar}}\right), 34.40\left(\mathrm{CH}_{2}\right), 31.92$ $\left(\mathrm{CH}_{2}\right), 29.67\left(2 \times \mathrm{CH}_{2}\right), 29.65\left(2 \times \mathrm{CH}_{2}\right), 29.60\left(\mathrm{CH}_{2}\right)$, $29.48\left(\mathrm{CH}_{2}\right), 29.35\left(\mathrm{CH}_{2}\right), 29.27\left(\mathrm{CH}_{2}\right), 29.18\left(\mathrm{CH}_{2}\right)$, $27.04(\mathrm{CH}), 25.06\left(\mathrm{CH}_{2}\right), 23.01\left(2 \times \mathrm{CH}_{3}\right), 22.68\left(\mathrm{CH}_{2}\right)$, $20.81\left(\mathrm{CH}_{3}-\mathrm{Ar}\right), 14.10\left(\mathrm{CH}_{3}\right)$.

\section{Thymyl Hexadecanoate (3r)}

Chromatographic purification gave colorless oil. $\mathrm{C}_{26} \mathrm{H}_{44} \mathrm{O}_{2}(M=388.63)$; yield $95.06 \%$; MS (EI): $\mathrm{m} / z(\%)$ $388\left(\mathrm{M}^{+}\right)$(5.8), 150 (100), 135 (65.4), 121 (4.3), 108 (3.4), 105 (4.0), 97 (3.3), 69 (5.6), 55 (6.1), 43 (4.3); RI (HP5-MS): 2772; ${ }^{1} \mathrm{H}$ NMR (500.13 MHz, $\mathrm{CDCl}_{3}$ ): $\delta$ (ppm) $7.20(1 \mathrm{H}, \mathrm{d}, J=8 \mathrm{~Hz}, \mathrm{Ar}-\mathrm{H}), 7.03(1 \mathrm{H}, \mathrm{d}, J=8 \mathrm{~Hz}$, Ar-H), $6.81(1 \mathrm{H}, \mathrm{s}, \mathrm{Ar}-\mathrm{H}), 2.98(1 \mathrm{H}, \mathrm{spt}, J=6.90 \mathrm{~Hz}$, $\mathrm{CH}), 2.58\left(2 \mathrm{H}, \mathrm{t}, J=7.5, \mathrm{CH}_{2}\right), 2.33\left(3 \mathrm{H}, \mathrm{s}, \mathrm{CH}_{3}\right), 1.78$ $\left(2 \mathrm{H}\right.$, quin, $\left.J=7.5 \mathrm{~Hz}, \mathrm{CH}_{2}\right), 1.51-1.28\left(24 \mathrm{H}, \mathrm{bm}, \mathrm{CH}_{2}\right)$, $1.21\left(6 \mathrm{H}, \mathrm{d}, J=6.90 \mathrm{~Hz}, \mathrm{CH}_{3}\right), 0.9(3 \mathrm{H}, \mathrm{t}, J=6.90 \mathrm{~Hz}$, $\left.\mathrm{CH}_{3}\right) ;{ }^{13} \mathrm{C}$ NMR $\left(125.76 \mathrm{MHz}, \mathrm{CDCl}_{3}\right): \delta(\mathrm{ppm}) 172.50$ $(\mathrm{C}=\mathrm{O}), 147.94\left(\mathrm{C}_{\mathrm{Ar}}\right), 136.99\left(\mathrm{C}_{\mathrm{Ar}}\right), 136.49\left(\mathrm{C}_{\mathrm{Ar}}\right), 126.99$ $\left(\mathrm{C}_{\mathrm{Ar}}\right), 126.33\left(\mathrm{CH}_{\mathrm{Ar}}\right), 122.73\left(\mathrm{C}_{\mathrm{Ar}}\right), 34.40\left(\mathrm{CH}_{2}\right), 31.92$ $\left(\mathrm{CH}_{2}\right), 29.68\left(2 \times \mathrm{CH}_{2}\right), 29.65\left(2 \times \mathrm{CH}_{2}\right), 29.60\left(\mathrm{CH}_{2}\right)$, $29.47\left(\mathrm{CH}_{2}\right), 29.35\left(\mathrm{CH}_{2}\right), 29.27\left(\mathrm{CH}_{2}\right), 29.19\left(\mathrm{CH}_{2}\right)$, $25.06\left(\mathrm{CH}_{2}\right), 23.01\left(2 \times \mathrm{CH}_{3}\right), 22.68\left(\mathrm{CH}_{2}\right), 20.81\left(\mathrm{CH}_{3}-\right.$ Ar), $14.10\left(\mathrm{CH}_{3}\right)$.

\section{Thymyl Heptadecanoate (3s)}

Chromatographic purification gave colorless oil. $\mathrm{C}_{27} \mathrm{H}_{46} \mathrm{O}_{2}(M=402.65)$; yield $72.50 \%$; MS (EI): $\mathrm{m} / \mathrm{z}(\%)$ $402\left(\mathrm{M}^{+}\right), 151$ (19.6), 150 (100), 136 (5.4), 135 (55.2), 109 (9.2), 71 (3.3), 69 (3.0), 57 (5.5), 55 (5.0), 43 (3.0); RI (HP5-MS): $2870 ;{ }^{1} \mathrm{H}$ NMR $\left(500.13 \mathrm{MHz}, \mathrm{CDCl}_{3}\right): \delta$ (ppm) $7.21(1 \mathrm{H}, \mathrm{d}, J=7.9 \mathrm{~Hz}, \mathrm{Ar}-\mathrm{H}), 7.03(1 \mathrm{H}, \mathrm{d}, J=7.9$ $\mathrm{Hz}, \mathrm{Ar}-\mathrm{H}), 6.82(1 \mathrm{H}, \mathrm{s}, \mathrm{Ar}-\mathrm{H}), 2.99(1 \mathrm{H}, \mathrm{spt}, J=6.80 \mathrm{~Hz}$, $\mathrm{CH}), 2.59\left(2 \mathrm{H}, \mathrm{t}, J=7.5, \mathrm{CH}_{2}\right), 2.33\left(3 \mathrm{H}, \mathrm{s}, \mathrm{CH}_{3}\right), 1.8$ $\left(2 \mathrm{H}\right.$, quin, $\left.J=7.5 \mathrm{~Hz}, \mathrm{CH}_{2}\right), 1.48-1.25\left(26 \mathrm{H}, \mathrm{bm}, \mathrm{CH}_{2}\right)$, $1.21\left(6 \mathrm{H}, \mathrm{d}, J=6.80 \mathrm{~Hz}, \mathrm{CH}_{3}\right), 0.91(3 \mathrm{H}, \mathrm{t}, J=6.90 \mathrm{~Hz}$, $\left.\mathrm{CH}_{3}\right) ;{ }^{13} \mathrm{C}$ NMR $\left(125.76 \mathrm{MHz}, \mathrm{CDCl}_{3}\right): \delta$ (ppm) 172.50 $(\mathrm{C}=\mathrm{O}), 147.9\left(\mathrm{C}_{\mathrm{Ar}}\right), 137.0\left(\mathrm{C}_{\mathrm{Ar}}\right), 136.5\left(\mathrm{C}_{\mathrm{Ar}}\right), 127.0\left(\mathrm{C}_{\mathrm{Ar}}\right)$, $126.30\left(\mathrm{CH}_{\mathrm{Ar}}\right), 122.70\left(\mathrm{C}_{\mathrm{Ar}}\right), 34.43\left(\mathrm{CH}_{2}\right), 31.96\left(\mathrm{CH}_{2}\right)$, $29.72\left(2 \times \mathrm{CH}_{2}\right), 29.52\left(2 \times \mathrm{CH}_{2}\right), 29.40\left(\mathrm{CH}_{2}\right), 29.31$ $\left(\mathrm{CH}_{2}\right), 29.22\left(\mathrm{CH}_{2}\right), 26.54\left(\mathrm{CH}_{2}\right), 25.09\left(\mathrm{CH}_{2}\right), 23.05(2 \times$ $\left.\mathrm{CH}_{3}\right), 22.72\left(\mathrm{CH}_{2}\right), 20.84\left(\mathrm{CH}_{3}-\mathrm{Ar}\right), 14.15\left(\mathrm{CH}_{3}\right)$.

\section{Thymyl Benzoate (3t)}

Chromatographic purification gave white solid. $\mathrm{C}_{17} \mathrm{H}_{18} \mathrm{O}_{2}(M=254.32)$; yield 95\%; MS (EI): $\mathrm{m} / \mathrm{z}(\%): 254$ $\left(\mathrm{M}^{+}\right),(9.7), 150$ (2.7), 149 (25.0), 133 (2.3), 106 (9.4), 105 (100), 91 (5.1), 78 (3.1), 77 (34.5), 51 (4.1); RI (HP5MS): $1955 ;{ }^{1} \mathrm{H}$ NMR $\left(500.13 \mathrm{MHz}, \mathrm{CDCl}_{3}\right): \delta$ (ppm) 8.26 
$(2 \mathrm{H}, \mathrm{d}, J=7.3 \mathrm{~Hz}, \mathrm{Ar}-\mathrm{H}), 7.68(1 \mathrm{H}, \mathrm{t}, \mathrm{Ar}-\mathrm{H}), 7.56(2 \mathrm{H}, \mathrm{t}$, Ar-H), $7.28(1 \mathrm{H}, \mathrm{d}, J=8 \mathrm{~Hz}, \mathrm{Ar}-\mathrm{H}), 7.11(1 \mathrm{H}, \mathrm{d}, J=8 \mathrm{~Hz}$, Ar-H), $6.98(1 \mathrm{H}, \mathrm{s}, \mathrm{Ar}-\mathrm{H}), 3.10(1 \mathrm{H}, \mathrm{spt}, J=6.90 \mathrm{~Hz}$, $\mathrm{CH}), 2.38\left(3 \mathrm{H}, \mathrm{s}, \mathrm{CH}_{3}\right), 1.25\left(6 \mathrm{H}, \mathrm{d}, J=6.90 \mathrm{~Hz}, \mathrm{CH}_{3}\right)$; ${ }^{13} \mathrm{C}$ NMR $\left(125.76 \mathrm{MHz}, \mathrm{CDCl}_{3}\right): \delta(\mathrm{ppm}) 165.33(\mathrm{C}=\mathrm{O})$, $148.11\left(\mathrm{C}_{\mathrm{Ar}}\right), 137.16\left(\mathrm{C}_{\mathrm{Ar}}\right), 136.62\left(\mathrm{C}_{\mathrm{Ar}}\right), 133.50\left(\mathrm{C}_{\mathrm{Ar}}\right)$, $130.12\left(2 \times \mathrm{CH}_{\mathrm{Ar}}\right), 129.63\left(\mathrm{CH}_{\mathrm{Ar}}\right), 128.60\left(2 \times \mathrm{CH}_{\mathrm{Ar}}\right)$, $127.15\left(\mathrm{CH}_{\mathrm{Ar}}\right), 126.45\left(\mathrm{CH}_{\mathrm{Ar}}\right), 122.84\left(\mathrm{CH}_{\mathrm{Ar}}\right), 27.24$ $(\mathrm{CH}), 23.03\left(2 \times \mathrm{CH}_{3}\right), 20.85\left(\mathrm{CH}_{3}-\mathrm{Ar}\right)$.

\section{3. Identification of Synthetized Compounds}

\section{3. 1. GC-MS Analysis}

MS spectra of samples of the synthesized compounds were recorded on a 7890/7000B GC/MS/MS triple quadrupole system (Agilent Technologies, USA, equipped with a Combi PAL auto sampler). The fused silica capillary column HP-5MS (5\% phenylmethylsiloxane, $30 \mathrm{~m} \times 0.25 \mathrm{~mm}$, film thickness $0.25 \mathrm{im}$, Agilent Technologies, Palo Alto, CA, USA) was used. The injector, source and interface operated at 250,230 and $300{ }^{\circ} \mathrm{C}$, respectively. The temperature program: from 60 for $5 \mathrm{~min}$ isothermal to $300{ }^{\circ} \mathrm{C}$ at a heating rate of $8{ }^{\circ} \mathrm{C} / \mathrm{min}$ and on $300{ }^{\circ} \mathrm{C}$ for $5 \mathrm{~min}$ isothermal. The solutions in hexane were injected in split ratio 10:1. The carrier gas was helium with a flow of $1.0 \mathrm{~mL} / \mathrm{min}$. Post run: back flash for 1.89 min, at $280^{\circ} \mathrm{C}$, with helium at $50 \mathrm{psi}$. MS conditions were as follows: ionization voltage of $70 \mathrm{eV}$, acquisition mass range $50-650$, scan time $0.32 \mathrm{~s}$. Semi-quantitative analysis was carried out directly from peak areas in the GC profile.

Linear retention indices (RI) were determined based on the retention times of $\mathrm{C}_{8}-\mathrm{C}_{40}$ alkanes run on HP-5MS column using the above mentioned temperature programme. ${ }^{41}$

\section{3. 2. NMR Analysis}

${ }^{1} \mathrm{H}$ NMR spectra were recorded in $\mathrm{CDCl}_{3}$ (isotopic enrichment 99.95\%) solutions at $25{ }^{\circ} \mathrm{C}$ using a Bruker AVANCE 500 instrument $\left(500.13 \mathrm{MHz}\right.$ for ${ }^{1} \mathrm{H}, 125.76$ $\mathrm{MHz}$ for ${ }^{13} \mathrm{C}$ ) using $5 \mathrm{~mm}$ inverse detection broadband probes and deuterium lock.

\section{4. Antimicrobial Activity}

\section{4. 1. Microbial Strains}

The in vitro antimicrobial activity of the synthesized compounds was tested against a panel of laboratory control strains belonging to the American Type Culture Collection Maryland, USA. Gram-positive: Bacillus subtilis ATCC 6633 and Staphylococcus aureus ATCC 6538; Gram-negative: Escherichia coli ATCC 8739, Pseudomonas aeruginosa ATCC 9027; fungal organisms: Aspergil- lus niger ATCC 16404 and Candida albicans ATCC 10231. The Gram-negative bacteria Salmonella abony NCTC 6017 and Salmonela typhimurium ATCC 14028 were obtained from the National Collection of Type Cultures. All microorganisms were maintained at $-20{ }^{\circ} \mathrm{C}$ under appropriate conditions and regenerated twice before use in the manipulations.

\section{4. 2. Screening of Antimicrobial Activity}

The minimal inhibitory concentration (MIC) of esters was determined based on a broth microdilution method in 96-well microtitre plates. ${ }^{42}$ The inocula of the bacterial strains were prepared from overnight broth cultures and suspensions were adjusted to $0.5 \mathrm{McF}$ arland standard turbidity. Dimethyl sulphoxide (10\% aqueous solution) was used to dissolve and to dilute samples to the highest concentration to be tested (stock concentrations $1 \mathrm{mg} / \mathrm{m}$ L). A serial doubling dilution of the samples was prepared in a 96-well microtiter plate, using the method of Sarker et al. ${ }^{43}$ with slight modifications. The minimal bactericidal/fungicidal concentration (MBC/MFC) was evaluated as the lowest concentration of tested samples at which inoculated microorganisms were $99.9 \%$ killed. Tests were carried out in triplicate.

\section{5. In silico Physico-chemical, Pharmacoki- netic and Toxicological Properties of the Synthetized Compounds}

In order to obtain a complete picture of the synthesized compounds 3a-t an in silico study was performed. Physico-chemical, pharmacokinetic and toxicological properties of compounds were calculated using the Molinspiration, ${ }^{44}$ admetSAR,${ }^{45}$ DataWarrior ${ }^{46}$ and Toxtree prediction tools. ${ }^{47}$

\section{Results and Discussion}

\section{1. Chemical Synthesis}

A small focused library of twenty thymyl esters was synthesized. To the best of our knowledge ten of twenty compounds are new (i.e. 3i, 3k-s; Scheme 1).

Although enzyme-catalyzed esterification of alcohols of different structures is a well-established approach, ${ }^{48}$ the enzymatic esterification of phenols is not frequently reported. ${ }^{49}$ Since a biocatalytic approach appeared especially appealing to us, we have tried to repeat the experimental protocol reported for the esterifcation of functionalized phenols with Candida antarctica lipase $(\mathrm{CAL}-\mathrm{B})^{49}$ using thymol and propanoic acid as substrates and tert-butyl methyl ether and hexane as solvents. However, although no reaction occurred in our hands, the biocatalytic approach deserves further investigation within the recent trends of green chemistry methodologies. 
a)<smiles>Cc1ccc(C(C)C)c(O)c1</smiles>

1<smiles>[R]C(=O)Oc1cc(C)ccc1C(C)C1C(=O)C(C)C1C(C)C([R])=O</smiles>

2 a-t
3 a-t

b)

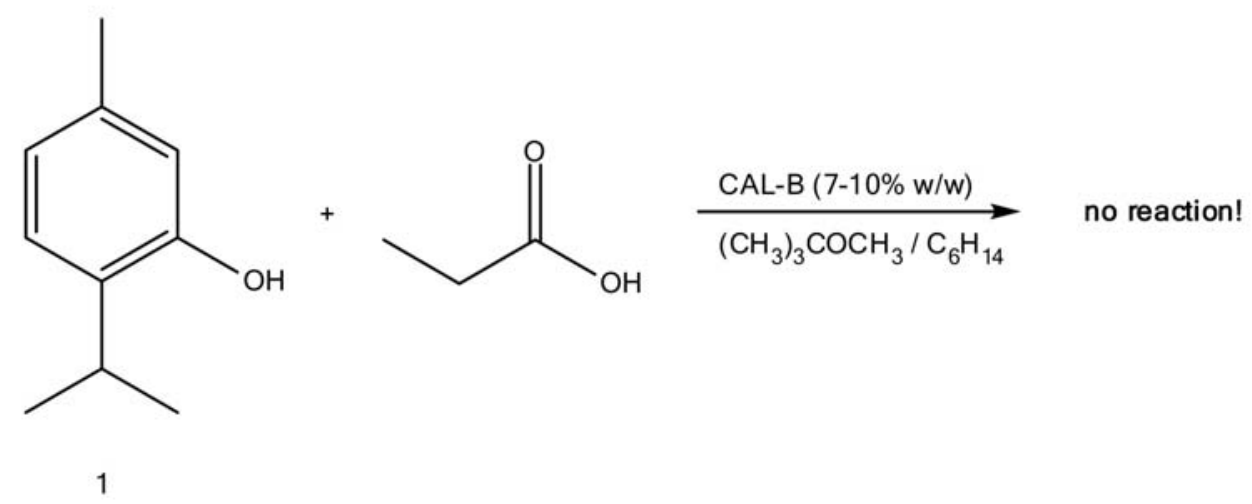

Scheme 2. Synthesis of thymyl esters: (a) chemical synthesis approach; (b) enzyme-catalyzed approach.

Table 1. Thymyl esters: chemical entity, yields (\%) and entry

\begin{tabular}{|c|c|c|c|}
\hline $\mathbf{R}$ & $\begin{array}{l}\text { Yield } \\
(\%)\end{array}$ & Product Name and Number & \\
\hline $\mathrm{H}_{3}$ & 85.00 & Thymyl Acetate & \\
\hline $\mathrm{CH}_{2} \mathrm{Cl}$ & 85.00 & Thymyl 2-Chloroacetate & \\
\hline $\mathrm{H}_{2} \mathrm{CH}_{3}$ & 82.00 & Thymyl Propanoate & \\
\hline $\mathrm{H}_{2} \mathrm{CH}_{2} \mathrm{CH}_{3}$ & 84.00 & Thymyl Butanoate & \\
\hline $\mathrm{H}\left(\mathrm{CH}_{3}\right)_{2}$ & 75.00 & Thymyl 2-Methylpropanoate & \\
\hline $\mathrm{H}_{2}\left(\mathrm{CH}_{2}\right)_{2} \mathrm{CH}_{3}$ & 87.00 & Thymyl Pentanoate & \\
\hline $\mathrm{H} C \mathrm{CH}(\mathrm{CH})$ & 81.00 & Methylbutanoate & \\
\hline$)_{3} \mathrm{CH}_{3}$ & 79.00 & & \\
\hline $\mathrm{CH}^{3}$ & 84.00 & eptanoate & \\
\hline$H^{2}$ & 85.00 & ctanoate & \\
\hline $\mathrm{CH}_{2}\left(\mathrm{CH}_{2}\right)_{6} \mathrm{CH}_{3}$ & 79.80 & ate & \\
\hline$x^{2}$ & 75.50 & & \\
\hline $\mathrm{CH}^{\circ}$ & 82.00 & Undecanoate & \\
\hline $\mathrm{CH}_{2}(\mathrm{C}$ & 76.30 & Dodecanoate & \\
\hline $\mathrm{CH}_{2}\left(\mathrm{CH}_{2}\right)_{10} \mathrm{CH}_{3}$ & 85.10 & Thymyl Tridecanoate & \\
\hline $\mathrm{H}_{2}\left(\mathrm{CH}_{2}\right)_{11} \mathrm{CH}_{3}$ & 72. & Tetradecan & \\
\hline I & 68.90 & Thymyl Pentadecanoate & \\
\hline $\mathrm{CH}_{2}(\mathrm{C}$ & 95.06 & Thymyl Hexadecanoate & \\
\hline $\mathrm{CH}_{2} \mathrm{C}$ & 72.50 & Thymyl Heptadecanoate & \\
\hline & 95.00 & Thymyl Benzoate & \\
\hline
\end{tabular}

\section{2. Antimicrobial Activity}

The results obtained in broth microdilution assay are presented in Supplementary data, Table S1. The assayed samples were less effective than antibiotic/antimycotic used as reference standard and if noted, activity was never greater than the values obtained for the parent compound 1 (MIC/MBC/MFC never exceeded $0.5 \mathrm{mg} / \mathrm{mL}$, Supplementary data, Table S1). The results are indicating selective susceptibility of the microorganisms, with $S$. aureus $(\mathbf{3 a}, \mathbf{b}, \mathbf{e}), P$. aeruginosa $(\mathbf{3} \mathbf{b}, \mathbf{j}, \mathbf{k}, \mathbf{p})$ and $C$. albicans $(\mathbf{3 a}-\mathbf{e}, \mathbf{g}, \mathbf{n}, \mathbf{p})$ being the most sensitive strains to synthesized derivatives. On the other hand, five microorganisms (B. subtilis, E. coli, S. abony, S. typhimurium and A. niger) were completely resistant to synthesized compounds tested (initial concentration $1 \mathrm{mg} / \mathrm{mL}$ ).

Five of our samples (3a,c,e, $, \mathbf{g}, \mathbf{t})$ are matching the samples tested by Mathela and collaborators, ${ }^{4}$ who were making evaluation of antibacterial activity on Streptococcus mutans (MTCC 890), S. aureus (MTCC 96), B. subtilis (MTCC 121), Staphylococcus epidermidis (MTCC 435) and E. coli (MTCC 723), and who reported the enhancement of the activity for esters in comparison to thymol. For all other synthesized compounds antimicrobial results are reported for the first time. An interesting fact is that MIC values by Mathela ${ }^{4}$ were three- (2-methylpropanoate $3 \mathbf{c}$ and 3-methylbutanoate $3 \mathbf{e}$ ) to even ten- (acetate 3a) times lower for $B$. subtilis than for thymol. Having in best case comparable, but never greater MIC values than for thymol itself, in our in vitro experiment we could not confirm such results. ${ }^{4}$ The importance of free hydroxyl group in the phenolic structure was confirmed in terms of 
activity when carvacrol was compared to its methyl ether, ${ }^{50}$ however results presented by Mathela ${ }^{4}$ are contrary to the above-mentioned fact and to our results (Supplementary data, Table S1).

\section{3. In silico Study}

\section{3. 1. Physico-chemical Properties of the Thymyl Esters 3a-t}

Lipinski's rule of 5 gives evaluation to drug-likeness and determines if a substance with certain pharmacological or biological activity has properties that would make it a likely orally active drug in humans. ${ }^{51}$ Calculations of important molecular parametars stated by Lipinski such as fragment based contributions and correction factors $\left(\mathrm{m}_{\mathrm{i}} \log \mathrm{P}\right)$, topological polar surface area (TPSA) and drug-likeness were obtained by Molinspiration software. ${ }^{44}$ Drug-likeness score of compounds $\mathbf{3 a}-\mathbf{t}$ is given in Suplementary data, Table S2.

Seven compounds $(\mathbf{3 a}-\mathbf{g})$ had $\mathrm{m}_{\mathrm{i}} \log \mathrm{P}$ values below 5 and thus were predicted to have sufficient oral bioavailability. The rest of the compounds were estimated as lypophilic. All of the tested compounds (3a-t) had TPSA below $60 \AA^{2}$, and thus were predicted to have both good intestinal absorption and good BBB penetration. All of the tested compounds ( $\mathbf{3 a}-\mathbf{t})$ had less than $10 \mathrm{H}$-bond acceptors $\left(\mathrm{n}_{\mathrm{ON}}\right)$ and less than $5 \mathrm{H}$-bond donors $\left(\mathrm{n}_{\mathrm{OHNH}}\right)$. The conformational flexibility, described by the number of rotatable bonds $\left(\mathrm{n}_{\text {rotb }}\right)$, for 12 compounds $(\mathbf{3 a}-\mathbf{k}, \mathbf{t})$ was between 0 and 10, which warrants good oral bioavailability. Finally, all compounds (3a-t) had both molecular weight (MW) and molecular volume below 500 .

Calculated physico-chemical properties showed that seven thymyl esters $(\mathbf{3 a}-\mathbf{g})$ were predicted to have good oral bioavailability, with values for fragment based contributions and correction factors $\mathrm{m}_{\mathrm{i}} \log \mathrm{P}<5$, TPSA $<140$, $\mathrm{MW}<500, \mathrm{n}_{\mathrm{ON}}<10, \mathrm{n}_{\mathrm{OHNH}}<5$ and $\mathrm{n}_{\text {rotb }}<10$.

\section{3. 2. Pharmacokinetic Properties of the Thymyl Esters 3a-t}

Absorption properties of compounds $\mathbf{3 a}-\mathbf{t}$ were predicted by admetSAR ${ }^{45}$ (Supplementary data, Table S3). The results suggested that all of the tested compounds (3a-t) might be able to pass through blood-brain barrier (BBB) and penetrate into the CNS, might be capable of being absorbed by intestine, and were supposed to have positive Caco-2 permeability. Moreover, compounds 3a-t were predicted as non-substrates for P-glycoprotein, noninhibitors of P-glycoprotein, and as non-inhibitors against renal organic cation transporter (ROCT).

Metabolic properties of the thymyl esters $3 \mathbf{a}-\mathbf{t}$ were predicted by admetSAR ${ }^{45}$ (Supplementary data, Table S4). None of the compounds was predicted as CYP450 2C9 and 2D6 substrate, while 16 compounds (3b,d,f,h-t) were predicted as CYP450 3A4 substrates. All of the te- sted compounds were predicted as CYP450 1A2 inhibitors, but none of them was predicted as CYP450 2D6 and 3A4 inhibitors. Moreover, 15 compounds $(\mathbf{3 b}, \mathbf{d}, \mathbf{h}-\mathbf{t})$ might be able to inhibit CYP450 2C19 enzyme, while only compound $3 \mathbf{t}$ was predicted as CYP450 2C9 inhibitor. Almost all thymyl esters (3a-s) were predicted to have low CYP inhibitory promiscuity, except compound $\mathbf{3 t}$.

\section{3. 3. Toxicological Properties of the Thymyl Esters 3a-t}

The structural alerts for DNA and protein binding for compounds 3a-t were predicted using Toxtree prediction tool based on decision tree approach.$^{47}$ Compounds 3a-t showed structural alerts for DNA binding as they were predicted as compounds able to undergo Michael addition. Moreover, compounds 3a-t showed structural alerts for protein binding due to their predicted ability to undergo Michael addition, ability to participate in acyl transfer and ability to undergo $\mathrm{S}_{\mathrm{N}} 2$ reactions (results given in Supplementary data, Table S5).

Toxicological properties of compounds $3 \mathbf{a}-\mathbf{t}$ predicted by admetSAR ${ }^{45}$ have characterized compounds $3 \mathbf{a}-\mathbf{t}$ as weak HERG (human Ether-à-go-go-Related Gene) inhibitors, non-AMES toxic and non-carcinogens, but highly toxic for fish, Tetrahymena pyriformis and honey bee. Ready biodegradable were supposed to be six compounds $(\mathbf{3 a}, \mathbf{c}-\mathbf{g})$. Depending on the risk for acute oral toxicity, only compound $\mathbf{3 b}$ was predicted as Category II, or compound with $\mathrm{LD}_{50}$ value greater than $50 \mathrm{mg} / \mathrm{kg}$ but less than $500 \mathrm{mg} / \mathrm{kg}$, while the rest of the tested compounds $(\mathbf{3 a}, \mathbf{c}-\mathbf{t})$ were predicted as Category III, or compounds with $\mathrm{LD}_{50}$ values greater than $500 \mathrm{mg} / \mathrm{kg}$ but less than $5000 \mathrm{mg} / \mathrm{kg}$. According to the $\mathrm{TD}_{50}$ values, compounds 3a-t were predicted as $»$ non-required $«$ or non-carcinogenic chemicals (Supplementary data, Table S6).

Only one study involving thymyl esters was undertaken to establish the potential acute toxicity in animal models. ${ }^{29}$ The esters shown no acute toxicity to mice at doses higher than $5000 \mathrm{mg} / \mathrm{kg}$ which represents good congruence and indicate the usefulness of data obtained in our in silico study.

Toxicological properties of thymyl esters $\mathbf{3 a}-\mathbf{t}$ predicted by DataWarrior ${ }^{46}$ have shown that only compound $\mathbf{3 b}$ has a high risk for all mutagenic, tumorigenic, reproductive and irritant effects. Compound $\mathbf{3 h}$ was predicted to have high risk for tumorigenic and irritant effects. However, all compounds were predicted to have high risk for irritant effects (Supplementary data, Table S7).

\section{Conclusion}

We synthesized twenty esters of thymol, of which ten represent new compounds. All of the compounds were employed in antimicrobial bioassay and was found that 
lower representatives of the synthesized homologous series of esters are antimicrobials comparable to thymol and can be considered as activity key holders, too. Results of our in silico study predicted that seven esters (lower representatives and short-chain fatty acids esters $\mathbf{3 a}-\mathbf{g}$ ) obey Lipinski's rule of five, showing drug-likeness. The rest of the compounds were estimated as lipophilic. All compounds, except thymyl 2-chloroacetate (3b) and thymyl hexanoate (3h), were predicted as non-mutagenic, non-tumorigenic, non-AMES toxic and non-carcinogenic, but highly toxic for fish, T. pyriformis and honey bee. They are likely to be absorbed by intestine and were predicted as ready biodegradable, weak HERG inhibitors, Category III of risk for acute toxicity, with no risk for reproductive effects, but with high risk for irritant effects. Taking in consideration predicted in silico properties and estimated drug likeness score, pharmacological and toxicological profile, thymyl esters might be used as prodrugs. Among the chemical bonds used to link parental drug and carrier, esters have already proven to be promising due to their amenability to hydrolysis in vivo and are most frequently used in order to enhance the lipophilicity ${ }^{52}$ and passive membrane transport.

\section{Acknowledgement}

The authors acknowledge the Ministry of Science and Technological Development of Serbia for financial support (project 451-03-821/2012-14 and 172044) as well as internal project of Faculty of Medicine, University of Niš (project no. 4).

\section{References}

1. E. Breitmaier, Terpenes: flavors, fragrances, pharmaca, pheromones, Wiley-VCH Verlag $\mathrm{GmbH} \& \mathrm{Co}$. KGaA, Weinheim, Germany, 2006.

2. J. R. Coats, L. L. Karr, C. D. Drewas, in: Naturally occurring pest bioregulators; P. H. Hedin, Ed.; ACS Symposium Series 449; American Chemical Society: Washington, DC, 1991, pp. 305 .

3. R. Tsao, S. Lee, P. J. Rice, C. Jensen, J. R. Coats, in Synthesis and Chemistry of Agrochemicals IV; D. R. Baker, J. G. Fenyes, G. S. Basarab, Eds; ACS Symposium Series 584; American Chemical Society: Washington, DC, 1995, p. 312.

4. C. S. Mathela, K. Singh, V. K. Gupta, Acta Pol. Pharm. 2010, 67, 375-380.

5. P. P. Kumbhar, P. M. Dewang, J. Sci. Ind. Res. 2001, 60, 645-648.

6. A. J. Polouse, R. Croteau, Arch. Biochem. Biophys. 1978, 187, 307-314. https://doi.org/10.1016/0003-9861(78)90039-5

7. K. Palaniappan, R. A. Holley, Int. J. Food Microbiol. 2010, 140, 164-168. https://doi.org/10.1016/j.ijfoodmicro.2010.04.001

8. A. Aijaz, K. Amber, Y. Snowber, A. K. Luqman, Fitoterapia, 2010, 81, 1157-1162.

https://doi.org/10.1016/j.fitote.2010.07.020

9. N. Mezzoug, A. Elhadri, A. Dallouh, S. Amkiss, N. S. Skali, J. Abrini, A. Zhiri, D. Baudoux, B. Diallo, M. El Jaziri, M. Idaomar, Res.-Gen. Tox. En. Mutat. 2007, 629, 100-110. https://doi.org/10.1016/j.mrgentox.2007.01.011

10. A. Andersen, Int. J. Toxicol. 2006, 25, 29-127. https://doi.org/10.1080/10915810600716653

11. J. Sikkema, J. A. M. DeBont, B. Poolman, Microbiol. Rev.1995, 59, 201-222.

12. I. M. Helander, H. L. Alakomi, K. Latva-Kala, T. MattilaSandholm, I. Pol, E. J. Smid, L. G. M. Gorris, A. Von Wright, J. Agric. Food Chem. 1998, 46, 3590-3595. https://doi.org/10.1021/jf980154m

13. R. J. W. Lambert, P. N. Skandamis, P. J. Coote, G. J. E. Nychas, J. Appl. Microbiol. 2001, 91, 453-462. https://doi.org/10.1046/j.1365-2672.2001.01428.x

14. S. E. Walsh, J. Y. Maillard, A. D. Russell, C. E. Catrenich, D. L. Charbonneau, R. G. Bartolo, J. Appl. Microbiol. 2003, 94 , 240-247. https://doi.org/10.1046/j.1365-2672.2003.01825.x

15. J. Xu, F. Zhou, B. P. Ji, R. S. Pei, N. Xu, Lett. Appl. Microbiol. 2008, 47, 174-179. https://doi.org/10.1111/j.1472-765X.2008.02407.x

16. M. Hyldgaard, T. Mygind, R. L. Meyer, Front. Microbiol. 2012, 3, 1-24.

https://doi.org/10.3389/fmicb.2012.00012

17. M. A. Ghannoum, L. B. Rice, Clin. Microbiol. Rev. 1999, 12, 501-517.

18. M. D. Cristani, M. Arrigo, G. Mandalari, F. Castelli, M. G. Sarpietro, D. Micieli, V. Venuti, G. Bisignano, A. Saija, D. Trombetta, J. Agric. Food Chem. 2007, 55, 6300-6308. https://doi.org/10.1021/jf070094x

19. A. Ahmad, A. Khan, F. Akhtar, S. Yousuf, I. Xess, L. Khan, N. Manzoor, Eur.J. Clin.Microbiol. Infect. Dis. 2012, 30, 41-50. https://doi.org/10.1007/s10096-010-1050-8

20. F. Bohlmann, P. K. Mahanta, J. Jakupovic, R. C. Rastogi, A. A. Natu, Phytochemistry 1978, 17, 1165-1172. https://doi.org/10.1016/S0031-9422(00)94308-5

21. F. Bohlmann, C. Zdero, Phytochemistry 1977, 16, 12431245. https://doi.org/10.1016/S0031-9422(00)94366-8

22. J. Zhao, Y. Li, Q. Lui, K. Gao, Food Chem. 2010, 120, 512-516. https://doi.org/10.1016/j.foodchem.2009.10.045

23. T. Xu, M. Gherib, C. Bekhechi, F. Atik-Bekkara, H. Casabianca, F. Tomi, J. Casanova, A. Bighellia, Flavour Fragr. J. 2015, 30, 83-90. https://doi.org/10.1002/ffj.3223

24. A. Talavera-Aleman, G. Rodriguez-Garcia, Y. Lopez, H. A. Garcia-Gutierrez, J. M. Torres-Valencia, R. E. del Rio, C. M. Cerda-Garcia-Rojas, P. Joseph-Nathan, M. A. Gomez-Hurtado, Phytochem. Rev. 2016, 15, 251-277. https://doi.org/10.1007/s11101-015-9412-6

25. A. Stojakowska, B. Kedzia, W. Kisiel, Fitoterapia 2005, 76, 687-690. https://doi.org/10.1016/j.fitote.2005.05.003 
26. S. Tawata, S. Taira, N. Kobamoto, M. Ishihara, S. Toyama, J. Pestic. Sci. 1996, 21, 141-146. https://doi.org/10.1584/jpestics.21.141

27. H. Liang, F. Bao, X. Dong, R. Tan, C. Zhang, Q. Lu, Y. Cheng, Molecules, 2007, 12, 1606-1613. https://doi.org/10.3390/12081606

28. J. Mastelić, I. Jerković, I. Blažević, M. Poljak-Blaži, S. Borovicì, I. Ivančić-Baće, V. Smrečki, N. Zarković, K. BrčićKostić, D. Vikić-Topić, N. Muller, J. Agric. Food Chem. 2008, 56, 3989-3996.

29. G. Angeles-Lopez, A. Perez-Vasquez, F. Hernandez-Luis, M. Deciga-Campos, R. Bye, E. Linares, R. Mata, J. Ethnopharmacol. 2010, 131, 425-432.

https://doi.org/10.1016/j.jep.2010.07.009

30. S. M. de Morais, N. S. Vila-Nova, C. M. Leal Bevilaqua, F. C. Rondon, C. H. Lobo, A. de Alencar Araripe Noronha Moura, A. D. Sales, A. P. Ribeiro Rodrigues, J. R. de Figuereido, C. C. Campello, M. E. Wilson, H. F. de Andrade Jr, Bioorg. Med. Chem. 2014, 22, 6250-6255.

https://doi.org/10.1016/j.bmc.2014.08.020

31. S. Robledo, E. Osorio, D. Munoz, L. M. Jaramillo, A. Restrepo, G. Arango, I. Velez, Antimicrob. Agents Chemother. 2005, 49, 1652-1655. https://doi.org/10.1128/AAC.49.4.1652-1655.2005

32. C. Bustos-Brito, M. Sanchez-Castellanos, B. Esquivel, J. S. Calderon, F. Calzada, L. Yepez-Mulia, A. Hernandez-Barragan, P. Joseph-Nathan, G. Cuevas, L. Quijano, J. Nat. Prod. 2014, 77, 358-363. https://doi.org/10.1021/np400964w

33. J. Grodnitzky, J. R. Coats, J. Agric. Food Chem. 2002, 50, 4576-4580. https://doi.org/10.1021/jf0201475

34. T. Yoshida, K. Mori, G. He, Heterocycles 1995, 41, 1923-1926. https://doi.org/10.3987/COM-95-7148

35. S. Wagner, A. Suter, I. Merfort, Planta Med. 2004, 70, 897-903. https://doi.org/10.1055/s-2004-832613

36. D. H. More, N. S. Pawar, P. M. Dewang, S. L. Patil, P. P. Mahulikar, Russ. J. Gen. Chem. 2004, 74, 217-218. https://doi.org/10.1023/B:RUGC.0000025504.59745.09

37. H. C. Brown, J. Am. Chem. Soc. 1938, 60, 1325-1328. https://doi.org/10.1021/ja01273a014

38. W. G. Rose, J. Am. Chem. Soc. 1947, 69, 1384-1387. https://doi.org/10.1021/ja01198a043
39. J. Paolini, A. Muselli, A. F. Bernardini, A. Bighelli, J. Casanova, J. Costa, Flavour Fragr. J. 2007, 22, 479-487. https://doi.org/10.1002/ffj.1824

40. D. Pavlović, B. Modec, D. Dolenc, Acta Chim. Slov. 2015, 62, 362-370. https://doi.org/10.17344/acsi.2014.1306

41. H. Van Den Dool, P. D. Kratz, J. Chromatogr. A 1963, 11, 463-471. https://doi.org/10.1016/S0021-9673(01)80947-X" Đt

42. NCCLS, Performance Standards for Antimicrobial Susceptibility Testing: Eleventh Informational Supplement, M100S11. 2003, National Committee for Clinical Laboratory Standards, Wayne, PA.

43. S. A. Sarker, L. Nahar Y. Kumarasamy, Methods 2007, 42, 321-324. https://doi.org/10.1016/j.ymeth.2007.01.006

44. Molinspiration Home Page. http://www.molinspiration.com/ (Accessed January 2017).

45. admetSAR Home Page. http://lmmd.ecust.edu.cn:8000/predict/ (Accessed January 2017).

46. DataWarrior Home Page. (Accessed January 2017).

47. G. Patlewicz, N. Jeliazkova, R. J. Safford, A. P. Worth, B. Aleksiev, Environ. Res. 2008, 19, 495-524.

48. E. Santaniello, P. Ferraboschi, P. Grisenti, Enzyme Microb. Technol. 1993, 15, 367-382. https://doi.org/10.1016/0141-0229(93)90123-J

49. G. J. H. Buisman, C. T. W. van Helteren, G. F. H. Kramer, J. W. Veldsink, J. T. P Derksen and F. P. Cuperus, Biotechnol. Lett.1998, 20, 131-136. https://doi.org/10.1023/A:1005368222340

50. H. J. D. Dorman, S. G. Deans, J. Appl. Microbiol. 2000, 80, 308-316. https://doi.org/10.1046/j.1365-2672.2000.00969.x

51. C. A. Lipinski, F. Lombardo, B. W. Dominy, P. J. Feeney, Adv. Drug. Deliv. Rev. 2012, 64, 4-17. https://doi.org/10.1016/j.addr.2012.09.019

52. D. Yancheva, E. Cherneva, M. Quick, B. Mikhova, B. Shivachev, R. Nikolova, A. Djordjevic, M. Untergehrer, G. Jürgenliemk, B. Kraus, A. Smelcerovic, Acta Chim. Slov. 2015, 62, 689-699.

https://doi.org/10.17344/acsi.2015.1418

\section{Povzetek}

Z derivatizacijo izhodnih struktur terpenoidov lahko pogosto pripravimo nove spojine, ki imajo izboljšane biološke aktivnosti. Naravni fenolov derivat timol, ki biosintezno nastane po terpenski poti, predstavlja dobro znan biocid z močnim antimikrobnim delovanjem in različnimi drugimi terapevtskimi aktivnostmi. Namen naše študije je bil pripraviti majhno, fokusirano knjižnico dvajsetih timilnih estrov s pomočjo ene same modifikacije fenolne funkcionalne skupine v timolu. Deset izmed pripravljenih spojin je novih. Vsem sintetiziranim spojinam smo in vitro določili antimikrobne lastnosti. Drug pomemben aspekt naše študije pa je bila uporaba in silico računskih metod za določitev fizikalno-kemijskih, farmakokinetičnih in toksikoloških lastnosti spojin, kar je omogočilo dodaten vpogled v njihove aktivnosti in daje nove usmeritve za nadaljnje raziskave. 